\title{
Ecological and soil hydraulic implications of microbial responses to stress - A modeling analysis
}

\author{
$\backslash$ begin $\{$ abstract $\}$
}

A better understanding of microbial dynamics in porous media may lead to improvements in the design and management of a number of technological applications, ranging from the degradation of contaminants to the optimization of agricultural systems. To this aim, there is a recognized need for predicting the proliferation of soil microbial biomass (often organized in biofilms) under different environments and stresses. We present a general multi-compartment model to account for physiological responses that have been extensively reported in the literature. The model is used as an explorative tool to elucidate the consequences of deploying microbial mechanisms characterized by the production of extracellular polymeric substances (EPS), the induction of cells into dormancy, and the allocation and reuse of resources between biofilm compartments. The mechanistic model is equipped with indicators allowing the microorganisms to monitor environmental and biological factors and react according to the current stress pressures. The feedbacks of biofilm accumulation on the soil water retention are also described. Model runs simulating substrate and water deprivation show that adaptive responses to the intensity and type of stress provide a clear benefit to microbial colonies. Results also demonstrate that the model may effectively predict qualitative patterns in microbial dynamics supported by empirical evidence, thereby improving our understanding of the effects of pore-scale mechanisms on the soil macroscale phenomena.

lend\{abstract\}

\section{Isection\{Introduction\}}

Almost all microorganisms on Earth are found in biofilms growing in soils and other surfaces of natural or manufactured origin \citep\{Vu2009\}. In most ecosystems, they grow under changing and often unfavorable conditions, which in soils, are characterized by relatively long periods of drying and rapid rewetting, accompanied by sharp changes in carbon availability. The evolutionary success of biofilms lies in their 
versatility to adapt to a wide range of environments, and their capacity to buffer, counteract or even benefit from external stresses. In general, biofilms provide a number of advantages to the embedded microbial colonies, increasing the hydration status, improving the efficiency of their digestive system, and reducing their mortality Icitep\{Flemming2010,Flemming2016,Or2007a\}. However, the mechanisms by which the microbial habitat is regulated and the potential impact of biofilm growth on porous media remain mostly unknown \citep\{Flemming2010,Or2007b\}. There is thus a need for understanding the response of biofilms to environmental conditions, with significant implications for the carbon cycle \citep\{Manzoni2012b\}, the degradation of contaminants \citep\{Marmonier2012\}, the design and management of technological and agricultural applications \citep\{DeJong2013,Thullner2010\}, and even the human life \citep\{Flemming2016,Selker1999\}.\\

The release of a polymeric matrix \citep[e.g.,][]\{Roberson1992,Tamaru2005\} and the capacity of organisms to be induced into dormancy \citep[e.g.,][]\{Konopka1999,Lennon2011\} are recognized as key microbial responses to stress. The biofilm matrix is a complex heterogeneous mixture of extracellular polymeric substances (EPS) of microbial origin resulting from deliberate secretions and lysis products. EPS constitute a variable proportion of the biofilm's total organic matter, ranging from $\$ 2 \$$ to $\$ 90 \backslash \% \$ \backslash$ citep $\{$ Chenu1995,Donlan2002,Flemming2010\}, consisting of polysaccharides, proteins, nucleic acids, lipids, and DNA Icitep\{Stoodley2002\}, and variable amounts of water \citep\{Or2007a\}. Their actual structure, quantity and composition are the result of the balance between needs and opportunities in a framework of biological and environmental factors Icitep\{Kim2010,Wilking2011\}. The nature of the matrix depends on the producer community and maturation stage \citep\{Flemming2016,Romani2008\}, but it is further determined by environmental conditions such as water flow rate, $\mathrm{pH}$, temperature, soil water content, and availability of nutrients/electron acceptors \citep\{Hand2008,More2014\}. Moreover, microorganisms may modulate the synthesis of EPS in response to such factors Icitep\{Chang2003,Redmile2015,Roberson1992,Sandhya2015\}, thereby counteracting the harmful effects of environmental stresses. EPS help maintaining cell functions and 
turgor, contribute to the uptake and assimilation of nutrients, and mediate cell adhesion to surfaces \citep\{Chenu1996,Flemming2010,Or2007a,Or2007b\}. In addition to the release of EPS, responses to stress include the induction of a reversible state of dormancy. When conditions are inappropriate, some microorganisms may change their functionality and slow down the metabolic processes in order to decrease their death rates \citep\{Brown1988,Stoodley2002\}.\\

Despite the benefits described above, these survival strategies entail side effects. First, the production of EPS involves significant investments of carbon, decreasing the amount of resources allocated to growth. Furthermore, the viability of natural and mutant non-producer strains \citep[e.g.,][]\{Seminara2012,Vandevivere1992a\} indicate that EPS are not essential for life. One could argue that evolution would not preserve organisms that produce functionless substances misusing energy and nutrients Icitep\{Chenu1995\}. Therefore, EPS should provide a competitive (or cooperative) advantage for the embedded microorganisms. A similar reasoning can be held for dormancy: despite inactive cells remain in a state of alertness, they cannot respond immediately when conditions improve Icitep[see][for details]\{Blagodatskaya2013\}. Cells must undergo a transition to recover their activity, potentially missing favorable periods. Therefore, dormancy could even be counterproductive in certain cases. Understanding the benefits of such cooperative behaviors frames the scope of this contribution. Difficulties mostly arise from the complex dynamics of the interactions and feedbacks between environmental and biological factors. \\

\section{\subsection\{The Impact of Biofilms on Pore-scale Conditions and Microbial Life\}}

The self-organization of microorganisms in biofilms results in a number of direct and indirect effects. First of all, the proliferation of biofilms in soils alters the hydraulic properties of porous media \citep\{Engesgaard2006,Rockhold2002,Volk2016\}, changing their capacity to transport and retain water and solutes. This effect emerges from the biofilm-soil interaction at the pore-scale and their own hydraulic attributes \citep\{Brangari2017\}. The most widely reported effect in bio-amended soils is bioclogging, i.e., a decrease in hydraulic conductivity due to EPS Icitep[e.g.,][]\{Thullner2002,Yarwood2006\} and/or cell accumulation 
\citep[e.g.,][]\{Vandevivere1992b,Wu1997\}. The reduction of water flow helps reducing detachment rates, preserving the hydration status and mitigating nutrient dilution and enzyme losses. However, under certain scenarios, the decrease in nutrient supply by impaired advection transport \citep\{Stewart2012, Thullner2002\} may turn dense biofilms into apparently undesired structures. $\ \backslash$

Bio-accumulation also enhances the retention of water Icitep\{Rosenzweig2012, Rubol2014\}, thereby improving the conditions in the vicinities of cells \citep\{Or2007b\}. Under wet conditions, the coating matrix forms an open structure that holds up many times its weight in water. When dried, the open structure shrinks, becoming a dense and amorphous frame that remains moist over a wide range of water potentials. This capacity depends on the morphology and distribution of the EPS matrix at the porescale \citep\{Brangari2017\}, but also on its composition. Along this line, Icitet\{Chenu1993\} found significant differences in the water retained by soils amended with different types of EPS of microbial origin, with a trend given as xanthan\$>\$EPS rhizobia\$>\$scleroglucan\$>\$dextran. \I

In addition to the enhancement of hydration, biofilm may contribute to the efficiency of the microorganisms' digestive system by increasing nutrient uptake rates in a number of ways. First, despite the fluxes through biofilms are lower than those for free water \citep\{Stewart2003\}, the increase in hydration modifies the advection-diffusion pathways \citep\{Chenu1996\}, with significant implications for substrate availability. Second, contact times and duration of periods suitable for growth or acclimation are lengthened \citep\{Or2007a\}. Third, biofilms act as a reservoir that retains nutrients, enzymes, and catalysis products close to the cells Icitep\{Flemming2010\}. Fourth, the matrix may behave as a molecular sieve sequestering dissolved and particulate substrate from the environment \citep\{Flemming2003,Flemming2016\}. Fifth, the carbon-rich EPS may be used as a source of carbon during periods of low resource availability. In contrast, when resources are abundant, microbes may allocate preferential resources to produce extra amounts of EPS Icitep\{Kakumanu2013,Roberson1992\}. Finally, despite being beyond the scope of this 
paper, EPS also alleviate the salt stress effect on cells \citep\{Sandhya2015\}, and facilitates their attachment to surfaces and to other cells \citep\{Vu2009\}.\\}

As a complement to these mechanisms, microbes may be induced to a reversible state of dormancy \citep[see][for details]\{Blagodatskaya2013\}. When conditions are not appropriate, dormant cells can persist for extended periods of time in a state of low to zero activity in which the mortality rate is lower than that for active cells. When conditions improve, inactive cells can gradually recover their functionality Icitep\{Stolpovsky2011\}. . In general, those organisms that are efficient at switching between states present advantages, particularly in those environments that experience recurrent and prolonged periods of stress |citep\{Konopka1999,Lennon2011\}.||

Hence, microorganisms can use, at least, two well-identified mechanisms to improve their fitness, increasing their capacity to replicate and survive in competitive environments. Responses might combine an improvement of the environmental characteristics with a reduction of the requirements for microbial preservation. The 'decision making' process is somehow similar to the quorum sensing mechanism of stimuli/response \citep\{Donlan2002,Parsek2005\}. Cells continuously interpret physicochemical signals (some of them released by themselves), which may regulate the deployment of these strategies. This way, microbial dynamics are modulated according to the nature and intensity of the stress \citep\{Chang2003,Sandhya2015\}. One could argue that such 'smart bioengineering' process ultimately promotes or mitigates the impact of biofilms on the soil to fulfill certain specific needs of the microbial community. $\ 1$

\section{\subsection\{Survival Strategies: Experimental Evidence and Modeling\}}

Due to the great importance of the aforementioned survival strategies on microbial proliferation, a large number of experimental and theoretical studies have been conducted. $\ \backslash$ 
Several experimental studies have pointed out that the release of EPS is modulated as a direct response to environmental stresses and microbial needs. \citet\{Roberson1992\} and \citet\{Chang2003\} found that biofilms growing under desiccation contain larger amounts of EPS. \citet\{Sandhya2015\} reported a significant increase in their production by bacteria exposed to high temperatures and salinity. \citet\{Tamaru2005\} found evidence supporting that these EPS help maintaining cellular viability under deep desiccation. \citet\{More2014\}, \citet\{Redmile2015\} and Icitet\{Roberson1992\} identified the EPS as a pool for resource allocation. Despite this compelling evidence, most modeling approaches do not include EPS responses to stressors. Most models focusing on bioclogging at the pore-scale do not differentiate between cells (responsible of activity) and other types of attached biomass (inactive but having other properties)

\citep[e.g.,][]\{Ezeuko2011,Pintelon2012,Rosenzweig2014,Soleimani2009,Thullner2008

\}. Some other models are written in terms of various microbial compartments \citep[e.g.,][]\{Aquino2008,Laspidou2002a,Laspidou2004a\}. Often, the mass balance of EPS (or of total biomass in simpler approaches) is defined as a more or less complex scheme of growth, decay and detachment \citep[e.g.,][]\{Ezeuko2011,Pintelon2012,Rosenzweig2014\}. An early study included the direct transformation of cells to EPS \citep\{Hsieh1994\}, disassociating production from substrate use, which is fundamental for modeling resource reallocation. Other models included a metabolic pathway in which the EPS could be decomposed and reused by cells as labile carbon \citep[e.g.,][]\{Aquino2008,Laspidou2004a\}. It is also worth mentioning that \citet\{Maggi2007\} included the competition of bacteria for water and space through limitations in their activity. However, none of these models account for the full feedbacks between the EPS and the environmental conditions. The most notable gaps in the representation of these processes encompass: (i) the EPS modulation of stress; (ii) the link between cellular demands (increased during stress) and the EPS synthesis; (iii) the EPS reuse as a source of carbon when nutrient conditions are not appropriate; and (iv) the impact of EPS on hydration status and nutrient uptake rate. \I 
Regarding the effects of dormancy on survival, Icitet\{MartinezLavanchy2009\} measured significant alterations of the microbial activity as a response to changes in carbon and oxygen supply. Icitet\{Stolpovsky2011\} associated such phenomena to a massive inactivation under inappropriate conditions. In \citet\{Kaprelyants1993\}, cells exposed to starvation could recover their functionality after a long period of dormancy. Other studies found that dormant cells tend to accumulate in the deepest regions of the biofilms \citep\{Kim2009,Williamson2012\}, pointing out that transport limitations inside biofilm structures may induce dormancy even under apparently favorable conditions. The studies modeling the phenomenon of dormancy include approaches that control the switching between activity stages with different degrees of complexity \citep[e.g.,][]\{Konopka1999,Manzoni2014,Stolpovsky2011\}. These models, however, have not been tested in scenarios with complex interactions between the microbial pools and the environmental conditions. $\mid \backslash$

\section{\subsection\{Model Rationale and Objectives\}}

Despite the empirical evidence, relating the production of EPS and dormancy to environmental stresses, drivers and consequences with process-based models is still difficult. This is mainly because biofilms (and more broadly soils) can be seen as an emerging property of a complex system that encompasses nonlinear and interconnected biogeochemical processes in a high-dimensional phase space. To the best of our knowledge, theoretical studies have tackled only some aspects of this complexity, and there are no empirical studies characterizing all the elements and mechanisms involved. $\ \backslash$

The aim of this paper is then to shed some light on these complex microbial dynamics by means of a multi-compartment soil microbial mechanistic model named SMMARTS for Soil Microbial Model to Account for Responses To Stress. The model includes up to seven well-differentiated lumped compounds (compartments) that approximate and therefore simplify the behavior of carbon in soils. The dynamics of biomass synthesis, inactivation/reactivation, decay, and allocation and reuse of carbon among biofilm components are modulated according to biological needs and competition for space, water and substrate. To this aim, the model uses indicators that allow the 
microorganisms to monitor environmental variables and biofilm status and react accordingly, reproducing the 'smart bioengineering' that governs the proliferation of microbial communities. The feedbacks of biofilms on soil water retention and their consequences on the nutrient uptake capacity are described at the pore-scale. A model with such a complexity is necessary to: (i) quantify microbial growth by including the most relevant carbon fluxes at the biofilm level; (ii) include mechanisms to modulate microbial dynamics according to experimental observations, with emphasis on responses to environmental stress; and (iii) estimate the feedbacks of biofilms on the soil hydraulic properties and the overall environmental conditions.

The resulting general model is developed in order to facilitate the study of the patterns of biofilm dynamics in porous media at the micro/meso-scale. It can then be employed as an explorative tool to disentangle external (i.e., environmental) and internal (i.e., nonlinearities) drivers of biofilms and their responses to environmental conditions. After some simplifications, we use this still very complex approach to explore scenarios based on reasonable ranges of parameter values mapping microbial traits. In this way, the ecological benefits of these survival strategies are evaluated under substrate or water deprivation. \\The consequences of flow velocity through bio-amended soils is not included in this paper and it is left for future research. The impact of evapotranspiration rates on saturation is neglected. $\ \backslash$

\section{\section\{Materials and Methods\}}

\section{|subsection\{SMMARTS Development\}}

\section{Isubsubsection\{Model Compartments\}}

The heterotrophic biofilm is differentiated into four lumped compartments: active cells $(A C)$, dormant cells (DC), extracellular polymeric substances (EPS), and extracellular enzymes (EZ). The AC compartment consists of the functional microorganisms embedded within the biofilm (considering mainly attached cells but it may also include planktonic organisms) that lead the behavior of the entire microbial system. The DC represents the previously active microorganisms that have undergone a reversible inactive state. The EPS pool is composed of soluble compounds, macromolecules and particulate materials that constitute the microbial matrix. Finally, the EZ compartment 
is formed by extracellular agents of microbial foraging responsible for the decomposition/solubilization of complex compounds. \I

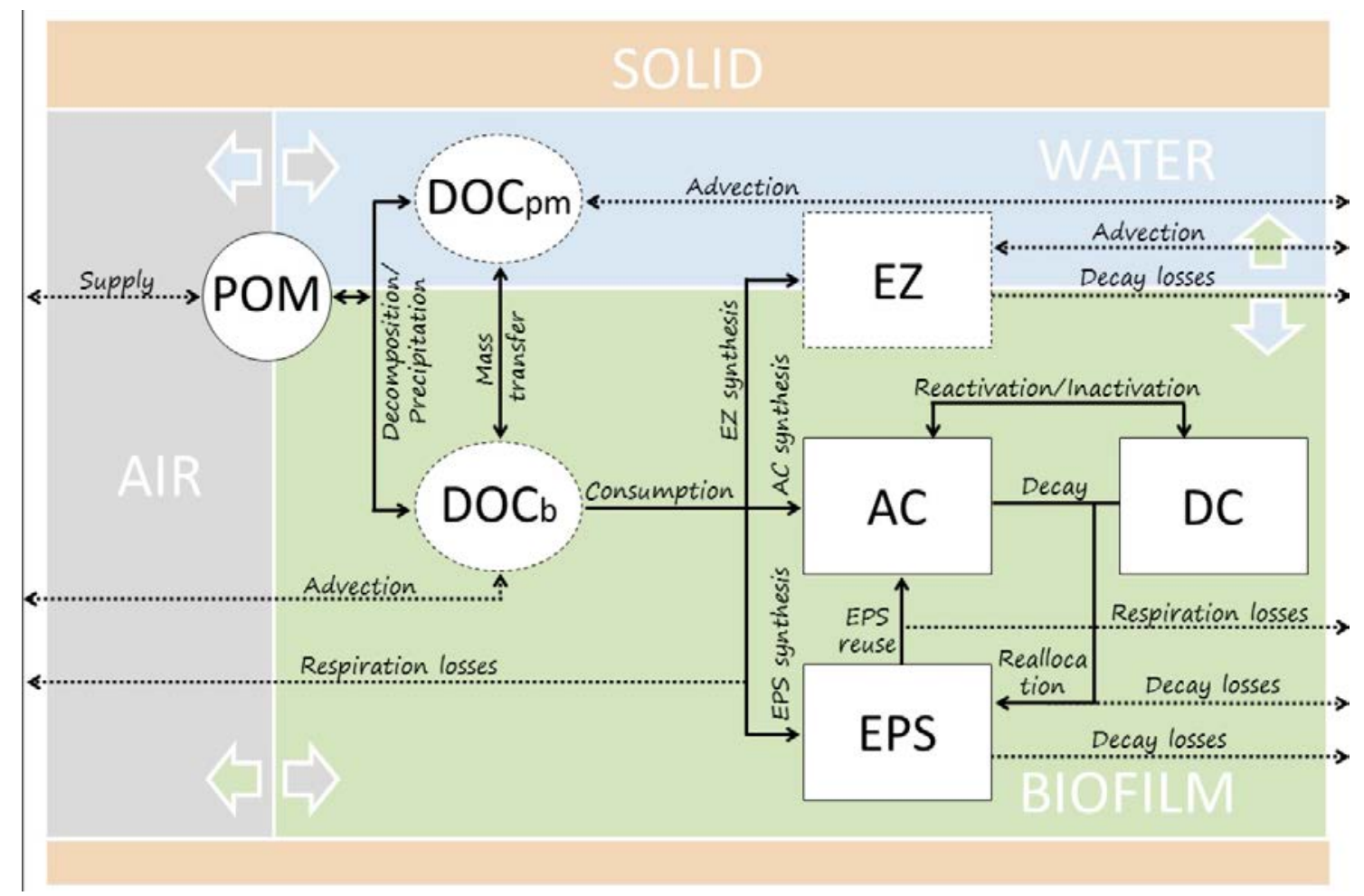

Icaption\{Sketch illustrating the biofilm, water, and air (of variable volume) inside a solid porous matrix and the biological processes described in the model. The solid arrows describe the fluxes between compartments, whereas the dotted arrows indicate those processes in which the system gains or loses carbon. The microbial compartments are differentiated into active cells ( $A C)$, dormant cells (DC), the polymeric matrix (EPS), and extracellular enzymes (EZ); and the substrate compartments into particulate organic matter (POM), dissolved organic matter in water (\$DOC_\{pm\}\$), and dissolved organic matter in biofilm phase (\$DOC_\{b\}\$). Each compartment is drawn on the phase(s) with which may be in contact (air, water or biofilm), as indicated by different shading. Dashed boxes represent compounds dissolved or in suspension. Granular material and air phase are considered passive with regard to the carbon cycle.\}

Biofilms require energy to proliferate. Here, for simplicity, we neglect the dynamics of nutrients such as nitrogen and phosphorus and of the electron acceptors like oxygen, albeit they can be easily incorporated. We thereby focus on conditions of carbon limitation that are prevalent in shallow mineral soils (C:N, C:P and C:O\$_\{2\}\$ ratios are generally lower than the critical ratios for inorganic nutrient immobilization). The nonmicrobial carbon compartments include: particulate organic matter (POM), dissolved organic matter in the pore-matrix water phase (\$DOC_\{pm\}\$), and dissolved organic matter inside the biofilm (\$DOC_\{b\}\$). $\backslash 1$ 
In this model, each compartment is assumed to be homogeneous in a specific control volume. The dynamics of the compartments are expressed as a system of mass balance equations (illustrated in Figure \refffigSM1\}). All model parameters are listed in Table 1.11

\section{\subsubsection\{Biofilm Effects on Water Distribution\}}

To account for the feedbacks between biofilm accumulation, hydraulic properties and environmental conditions, the distribution of water and biomass is defined at the pore-scale level based on the framework presented in \citet\{Brangari2017\}. Despite using the simple capillary tube analogy, this approach effectively reproduced saturation changes in bio-amended soils. The model considers a biofilm that may retain variable amount of water depending on suction. Such a volume of water in biofilms (\$\theta_\{b\}\$ [\$-\$]) may be defined from the water retention capacities of EPS with some spatial restrictions

$$
\theta_{b}=\min \left(\frac{\overline{E P S}}{\rho_{w}} a \psi^{-b}, \frac{c \overline{E P S}}{\rho_{w}}, \phi_{M}\right)
$$

where the overbar indicates the average concentration of a compartment (here the dry mass of EPS) over a unit volume of soil $[\$ M L \wedge\{-3\} \$], \$ \backslash r h o \_\{w\} \$[\$ M L \wedge\{-3\} \$]$ is the

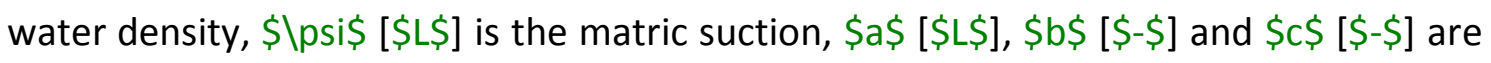
experimental parameters for the water retention capacity of EPS, and \$phi_\{M $\}$ [\$ is the maximum volume available, which is susceptible to be occupied by any of the phases studied. $\$

The first term in (\ref\{eqSM1\}) represents the shrinking/swelling capacity of the biofilm, which depends mainly on the composition of the EPS. Icitet\{Rosenzweig2012\} reported that $\$ a=105.76 \mathrm{~cm} \$$ and $\$ b=0.489 \$$ for pure xanthan (when suction is expressed in \$cm\$ of water), whereas \citet\{Chenu1993\} observed that dextran shows almost no retention capacity (\$a\rightarrow0\$). The impact of microbial cells on the soil water retention curve (SWRC) is here neglected to better isolate the effects of EPS, 
segregating the role of the cellular water in extracellular processes. The second term restricts the maximum volumetric density of EPS. In this line, Icitet\{Chenu1993\} observed that xanthan may retain up to $\$ 70 \$$ times its weight in water $(\$ c=70 \$)$. The third term constrains \$ $\backslash$ theta_\{b\}\$ to spatial and water limitations. In fully-hydrated porous media, \$phi_\{M\}\$ is equal to the effective porosity of the soil \$phi_\{ef\}\$ [\$\$], i.e., spatially restricted to the saturated minus the residual water content (\$\} \backslash p h i \_ \{ e f \} = \backslash \text { theta_\{s\}-\theta_\{r\}\$). Nevertheless, when water availability is limited, } the water-suction equilibrium assumed in the first part of (\ref\{eqSM1\}) might not be achieved and \$theta_\{b\}\$ fulfills specific water limitations (being \$ $\backslash p h i \_\{M\} \$$ the maximum amount of water available, defined from the soil suction in (\ref\{eqSM16\}), Section 2.2). 11

Biofilm bodies comprise complex structures that form pores, voids, and channels, which induce significant structural changes in the pore-matrix. Its accumulation therefore alters the size and geometry of the pore space, leading to indirect changes in the macroscopic retention properties. When pore-scale effects are integrated over the whole control volume, the volume of pore-matrix water $\$$ theta_\{pm\}\$ [\$-\$] retained in the open pore space not occupied by biofilm may be written as

$$
\theta_{p m}=\left[\phi_{e f}-\theta_{b}\right]\left[1+\alpha^{n} \psi^{n}\left[\frac{\phi_{e f}-\theta_{b}}{N \phi_{e f}}\right]^{\frac{n}{2}}\right]^{\frac{1}{n}-1}+\theta_{r},
$$

where $\$ n \$[\$-\$]$ and $\$ \backslash$ alpha $\left[\$ L^{\wedge}\{-1\} \$\right.$ ] are the experimental van Genuchten's parameters \citep\{vanGenuchten1980\} obtained with biofilm-free soils, and \$N\$ [\$-\$ ] is a theoretical parameter that accounts for the channeled architecture of biofilm. The larger is \$N\$, the more intricate is the morphology of the biofilm-soil system at the pore-scale, resulting in a potential increase in the water holding capacity. The reader is referred to \citet\{Brangari2017\} for derivation and details.

\section{\subsubsection\{Non-Microbial Carbon\}}

Carbon is used to synthetize new cells, enzymes and matrix compounds, and to produce energy for their maintenance. In our model scheme, carbon supplies are 
partitioned between particulate (POM) and dissolved forms (DOC) \citep[as in e.g.,][]\{Greskowiak2005b,Schimel2003\}; and the latter is further distinguished between $\$ D O C \_\{p m\} \$$ and $\$ D O C \_\{b\} \$$ Icitep[as in e.g.,][]\{Orgogozo2010\}. Such a differentiation is required to account for the relatively large differences in

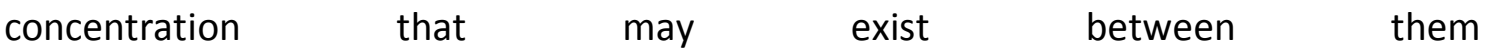
Icitep\{Billings2015,Stewart2003,Stewart2012\}. This scheme is simple, but may effectively represent the wide range of forms in which organic matter is found in ecosystems. \\

POM here includes diverse undissolved materials that range from the coarser fraction of the organic matter to molecules of complex composition. POM cannot be directly assimilated into microbial biomass, but requires an enzyme-mediated extracellular depolymerization step to be reduced to simpler dissolved compounds (here DOC) Icitep\{Romani2004\}. In porous materials, decomposition rates are constrained by the spatial limitations on solute diffusion, which depend on soil tortuosity. Such a process is also rate-limited by the solubility of DOC \cited[as in][]\{Greskowiak2005b\}. Here, \$DOC_\{eq\}\$ represents the value of saturation. The mass balance for POM in time (\$t\$, [\$T\$]) may then be expressed as

$$
\frac{\partial \overline{P O M}}{\partial t}=\underbrace{A D D}_{P O M \text { supply }}-\underbrace{\left[\frac{\theta_{b}}{\theta_{b}+\theta_{p m}} \xi_{\tau} \mu_{P O M} \xi_{P O M} \overline{E Z} \frac{D O C_{e q}-\overline{D O C_{b}}}{D O C_{e q}}+\frac{\theta_{p m}}{\theta_{b}+\theta_{p m}} \xi_{\tau} \mu_{P O M} \xi_{P O M} \overline{E Z} \frac{D O C_{e q}-\overline{D O C_{p m}}}{D O C_{e q}}\right]}_{\text {POM decomposition }}
$$

where $\$$ loverline $\{\mathrm{POM}\} \$\left[\$ \mathrm{ML}^{\wedge}\{-3\} \$\right]$ is the dry mass of POM per unit volume of soil, \$loverline $\{\mathrm{EZ}\} \$\left[\$ \mathrm{ML}^{\wedge}\{-3\} \$\right.$ is the dry mass of $\mathrm{EZ}$ per unit volume of water and biofilm,

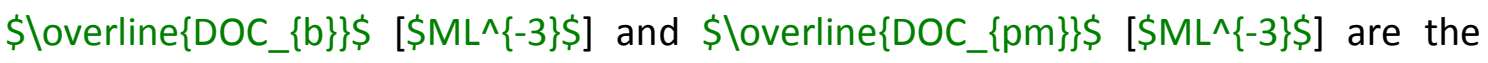
concentrations of $D O C$ in the respective pools, $\$ A D D \$\left[S L^{\wedge}\{-3\} T^{\wedge}\{-1\} \$\right.$ ] is the

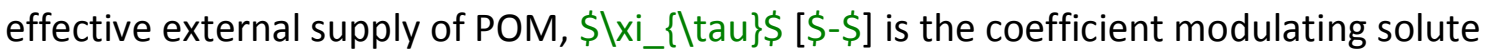
transfer, $\$ \backslash m u \_\{P O M\} \$\left[\$ T^{\wedge}\{-1\} \$\right]$ is the maximum specific decomposition rate, and

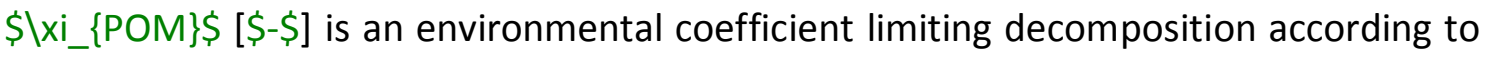
the amount of POM. The decomposition fluxes in (\ref\{eqSM3\}) are proportional to the enzyme concentration, and driven by the disequilibrium of DOC in the individual pools. 
The solute diffusion coefficient $\$ \mid x i \_\{\mid t a u\} \$$ for partially-saturated soils is based on Archie's second law for solute diffusivities \citep[see][for details]\{Hamamoto2010\}

$$
\xi_{\tau}=\phi_{e f}^{\Omega}\left[\frac{\theta_{b}+\theta_{p m}}{\phi_{e f}}\right]^{\gamma},
$$

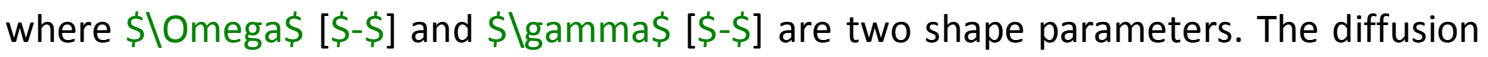
thresholds in the original Archie's law are assumed equal to \$0\$ in (\ref\{eqSM4\}). With this premise, decomposition rates are ensured even at low saturation (\$\|approx\theta_\{r\}\$) since water (solvent) and POM are considered to be "in contact" at the pore-scale. Note also that $\$ x i_{1}\{\mid \operatorname{tau}\} \$$ is the inverse of the tortuosity and depends on soil type by means of (\ref\{eqSM2\}). Yet, the reduced diffusivity through biofilm bodies \citep[described in e.g.,][]\{Or2007a\} is neglected. $\backslash \backslash$

\$ $\mid x i \_\{P O M\} \$$ is a coefficient that limits the effective decomposition rate as \$loverline $\{\mathrm{POM}\} \$$ decreases. It may be described by the generalized saturating function based on the Michaelis-Menten kinetic model

$$
\xi_{\mathrm{X}}=\frac{\overline{\mathrm{X}}}{\mathrm{X}_{K}+\overline{\mathrm{X}}}
$$

where $\$ \mid c h i \_\{K\} \$ \$ M L \wedge\{-3\} \$$ ] (here $\$ P O M \_\{K\} \$$ ) is the half-saturation constant of a generic variable \$Chi\$ (here POM). .।

Carbon decomposed in ( $\backslash$ ref $\{$ eqSM3\}) is then diverted to the DOC compartments. \$loverline $\left\{D O C \_\{p m\}\right\}$ and $\$ \backslash$ loverline $\left\{D O C \_\{b\}\right\}$ are affected by diverse and varying processes of transport, formation and consumption defined in ( $\backslash$ ref $\{$ eqSM6\}) and (\ref\{eqSM7\}) (Figure \refffigSM1\}). 


$$
\begin{aligned}
\frac{\partial \theta_{p m} \overline{D O C_{p m}}}{\partial t}= & \underbrace{\frac{\theta_{p m}}{\theta_{b}+\theta_{p m}} \xi_{\tau} \mu_{P O M} \xi_{P O M} \overline{E Z} \frac{D O C_{e q}-\overline{D O C_{p m}}}{D O C_{e q}}}_{\text {POM decomposition-DOC }}-\underbrace{v_{w}\left[\theta_{p m}-\theta_{r}\right] \frac{\partial \overline{D O C_{p m}}}{\partial z}}_{\text {Advection }} \\
& +\underbrace{\frac{\theta_{p m}}{\theta_{b}+\theta_{p m}} \xi_{\tau} D_{0} \frac{\partial^{2} \overline{D O C_{p m}}}{\partial \mathbf{z}^{2}}}_{\text {Diffusion }}-\underbrace{\Gamma}_{\text {Masstransfer }}
\end{aligned}
$$

$$
\begin{aligned}
& \frac{\partial \theta_{b} \overline{D O C_{b}}}{\partial t}=\underbrace{\frac{\theta_{b}}{\theta_{b}+\theta_{p m}} \xi_{\tau} \mu_{P O M} \xi_{P O M} \overline{E Z} \frac{D O C_{e q}-\overline{D O C_{b}}}{D O C_{e q}}}_{\text {OM decomposition-DOC }}-\underbrace{\mu_{C} \xi_{\phi, P} \xi_{D O C} \overline{A C}}_{\text {Consumption }}-\underbrace{v_{w} \theta_{b} \frac{\partial \overline{D O C_{b}}}{\partial z}}_{\text {Advection }} \\
& +\underbrace{\frac{\theta_{b}}{\theta_{b}+\theta_{p m}} \xi_{\tau} D_{0} \frac{\partial^{2} \overline{D O C_{b}}}{\partial z^{2}}}_{\text {Diffusion }}+\underbrace{\Gamma_{1}}_{\text {Masstransfer }}
\end{aligned}
$$

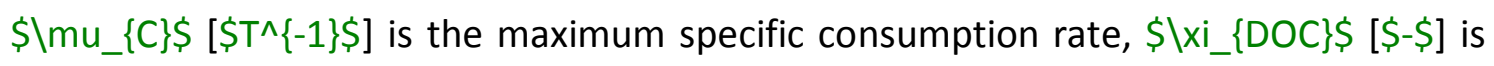
an environmental coefficient limiting consumption rates, $\$ \backslash x i \_\{\backslash p s i, P\} \$[\$-\$]$ is a

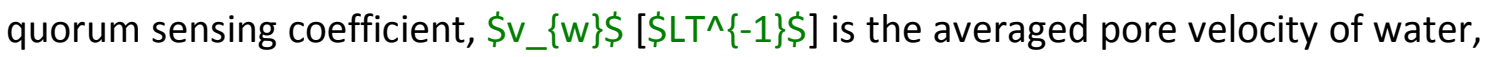

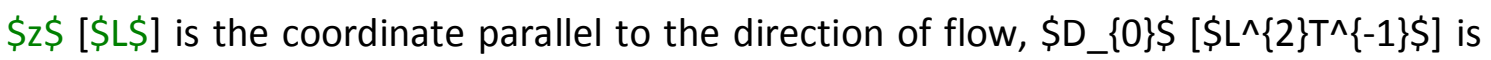
the solute diffusion coefficient in water, and $\$ \backslash G a m m a \$\left[M L \wedge\{-3\} T^{\wedge}\{-1\} \$\right.$ ] is the mass exchange function. \I

Consumption rates are mathematically modulated by the coefficients $\$ \backslash x i_{-}\{D O C\} \$$ and $\$ \backslash x i \_\{\mid p s i, P\} \$$. They simulate the microorganisms' capacity to monitor and respond to environmental and biological variables. The coefficient $\$ x i_{-}\{D O C\}$ consists in a nonlinear term defined in (\ref\{eqSM5\}), just replacing $\$ \backslash c h i \$$ by $\$ D O C \_\{b\} \$$. It can easily include growth limitation caused by electron acceptor shortage Icitep[as in][]\{Laspidou2002b,Rodriguez-Escales2014\}. Note that only \$DOC_\{b\}\$, which is in the immediate vicinity of microbial cells, is used for metabolic processes. The coefficient \$ \xi_\{ \psi,P\}\$ reduces carbon uptake when the microbial population is high,

$$
\xi_{\phi, \mathrm{P}}=\left[1-\frac{\overline{A C}+\overline{D C}}{\rho_{c} \phi_{e f}}\right]^{\beta_{P}},
$$


where $\$ \backslash$ overline $\{A C\} \$$ and $\$ \backslash$ verline $\{D C\} \$$ are the dry masses of $A C$ and $D C$ per unit

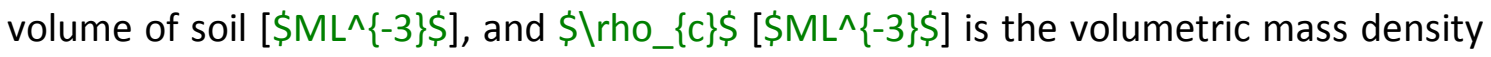
of the microbial cells. \$ $\backslash$ beta_ $\{P\} \$$ [\$-\$ $]$ is a shape factor that determines the strength of the population regulation. It ranges from $\$ 0 \$$ to $\$ \backslash$ infty for mechanistic rationale and defines the curvature of the inhibition expression. The resulting $\$ \mid x i \_\{\mid p s i, P\} \$$ tends to $\$ 1 \$$ (no inhibition) when the volume of cells is much smaller than the free space and decreases with increasing total microbial population (\$overline $\{A C\}+\backslash$ overline $\{D C\} \$$ ) as space becomes limiting. $\backslash \backslash$

Advection and diffusion terms in the mass blance equations for DOC represent powerful transport processes that may either provide or flush out dissolved carbon from the system. For simplicity, water velocity is considered phase independent (averaged and then assumed equal in open pores and in biofilms), and the effect of solute diffusion is linearized in (\ref\{eqSM6\} and \ref\{eqSM7\}). Note that, due to the similarity between process-regulators at the pore-scale, the effective diffusion is determined by \$Xi_\{\tau\}\$ (recall (\ref\{eqSM4\})).. \I

The mass transfer function $\$ \backslash G a m m a \$$ controls the flow of dissolved carbon between the two DOC compartments. It is driven by the difference in concentration, the size of the intricate surface of contact, and the advective-dispersive flow. The faster the exchange of mass, the faster the equilibrium is reached. When the characteristic time of consumption is much longer than that of the mass transfer (rates and contact areas are relatively large at the pore-scale), equilibrium can be assumed (\$\overline $\left\{D O C \_\{b\}\right\}==\backslash$ overline $\left\{D O C \_\{p m\}\right\} \$$ ).

\section{Isubsubsection\{Microbial Compartments\}}

The main carbon fluxes in biofilms represent the synthesis of new biomass, the production and reuse of EPS, the transition to and from the dormant state, the losses of different nature, and the reallocation of decay products. Accordingly, the mass balance equations describing the four microbial compartments are (Figure \ref\{figSM1\}) 


$$
\begin{aligned}
& \frac{\partial \overline{A C}}{\partial t}=\underbrace{Y\left[1-\lambda_{E P S}^{*}-\lambda_{E Z}^{*}\right] \mu_{C} \xi_{\phi, P} \xi_{D O C} \overline{A C}}_{A C \text { synthesis (DOC consumption) }}+\underbrace{Y \mu_{E P S} \xi_{E P S} \xi_{E Z}\left[1-\xi_{D O C}\right] \overline{A C}}_{A C \text { synthesis (EPS decomposition) }} \\
& -\underbrace{\tau_{i}\left[1-\xi_{D O C}\right] \xi_{\phi, \mathrm{P}} \overline{A C}}_{\text {Dormancy }}+\underbrace{\tau_{a}^{\xi_{D O C} \overline{D C}}}_{\text {Reactivation }}-\underbrace{K_{D}^{A C} \overline{A C}}_{\text {AC decay }} \\
& \begin{aligned}
\frac{\partial \overline{E P S}}{\partial t} & =\underbrace{Y \lambda_{E S}^{*} \mu_{C} \xi_{\phi, \mathrm{P}} \xi_{D O C} \overline{A C}}_{\text {EPS synthesis }(\text { DOC consumption })}-\underbrace{\mu_{E P S} \xi_{E P S} \xi_{E Z}\left[1-\xi_{D O C}\right] \overline{A C}}_{\text {EPS decomposition }}+\underbrace{\lambda_{D}\left[K_{D}^{A C} \overline{A C}+K_{D}^{D C} \overline{D C}\right]}_{\text {Decayed mass redistribution }}, \\
& -\underbrace{K_{D}^{E P S} \overline{E P S}}_{\text {EPS decay }},
\end{aligned}
\end{aligned}
$$$$
\frac{\partial \overline{D C}}{\partial t}=\underbrace{\tau_{i}\left[1-\xi_{D O C}\right] \xi_{\phi, \mathrm{P}} \overline{A C}}_{\text {Dormancy }}-\underbrace{\tau_{a} \xi_{D O C} \overline{D C}}_{\text {Reactivation }}-\underbrace{K_{D}^{D C} \overline{D C}}_{\text {DC decay }},
$$

$\frac{\partial\left[\theta_{b}+\theta_{p m}\right] \overline{E Z}}{\partial t}=\underbrace{Y \lambda_{E Z}^{*} \mu_{C} \xi_{\phi, P} \xi_{D O C} \overline{A C}}_{\text {EZ synthesis(DOC consumption) }}-\underbrace{K_{D}^{E Z}\left[\theta_{b}+\theta_{p m}\right] \overline{E Z}}_{\text {EZ decay }}-\underbrace{v_{w}\left[\theta_{b}+\theta_{p m}-\theta_{r}\right] \frac{\partial \overline{E Z}}{\partial \mathbf{z}}}_{\text {Advection }}+\underbrace{\xi_{\tau} D_{0} \frac{\partial^{2} \overline{E Z}}{\partial \mathbf{z}^{2}}}_{\text {Diffusion }}$

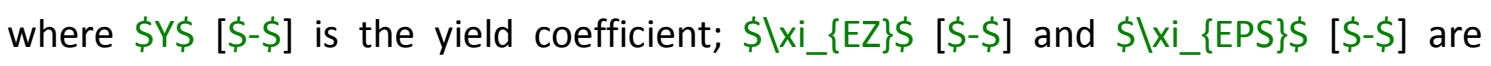
limiting environmental coefficients based on the requirements of EZ and EPS; $\$ \backslash \mid a m b d a \wedge\{*\} \_\{E Z\} \$[\$-\$]$ and $\$ \backslash \mid a m b d a \wedge\{*\} \_\{E P S\}$ [\$-\$] are effective coefficients of

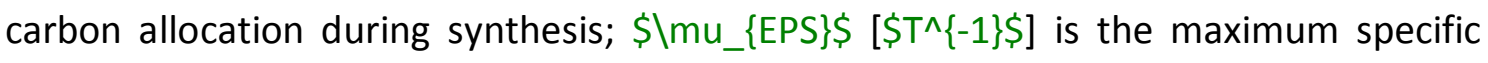

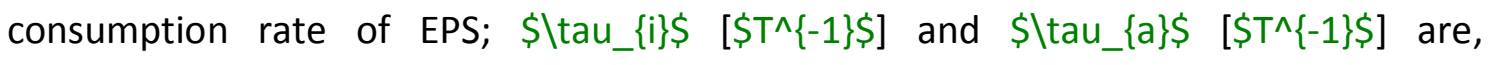
respectively, the maximum rates of inactivation and reactivation of cells; $\$ K$ _ $\{D\} \$$ $\left[\$ T^{\wedge}\{-1\} \$\right.$ ] are decay rate coefficients for the compartments indicated in the superscripts; and \$Vlambda_\{D\}\$ [\$-\$] is the decayed mass reallocation ratio. $\backslash 1$

The fraction $\$ Y \$$ of the carbon substrate taken up (not lost by respiration) is converted to new biomass. It is equivalent to the microbial carbon-use efficiency (CUE) when DOC is the only source of carbon (AC synthesis via EPS reuse is not taking place; see details below). The allocation coefficients $\$ \backslash / a m b d a \wedge\{*\} \_\{E P S\}[\$-\$]$ and $\$ \backslash l a m b d a \wedge\{*\} \_\{E Z\} \$$ [\$-\$] indicate the effective fraction of carbon diverted to produce EPS and EZ, respectively \citep[based on the formation coefficients described in][]\{Laspidou2004a\}. 
Different from previous approaches, the synthesis of EPS and EZ is modulated to improve the well-being of microorganisms according to environmental and biological variables. An effective use of carbon permits investing additional amounts of resources to AC when EPS or EZ requirements are low. Such adaptive modulations may be mathematically described by

$$
\begin{aligned}
& \lambda_{E P S}^{*}=\lambda_{E P S} \xi_{\phi, \theta_{b}}, \\
& \lambda_{E Z}^{*}=\lambda_{E Z}\left[1-\xi_{E Z}\right],
\end{aligned}
$$

where \$Vlambda_\{EPS\}\$ [\$-\$] and \$Vlambda_\{EZ\}\$ [\$-\$] are the maximum coefficients of carbon allocation, and $\$ x i \_\{E Z\} \$$ governs the release of EZ (again, obtained using

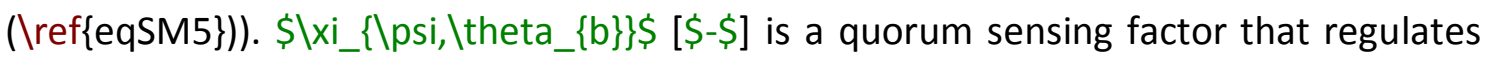
the production of EPS according to their concentration and water requirements as

$$
\xi_{\phi, \theta_{b}}=\left[1-\frac{\theta_{b}}{\phi_{e f}}\right]^{\beta_{\theta_{b}}} .
$$

\$ $\mid x i \_\{\backslash p s i, \backslash \text { theta_\{b\}\}\$ tends to } \$ 1 \$ \text { (no inhibition) when the volume of water in the }$ biofilm phase is much smaller than \$ $\backslash p h i \_\{e f\} \$$, and therefore the resulting effective ratio of allocation is approximately $\$ \backslash$ lambda_\{EPS\}\$. \$ $\backslash x i \_\{\mid p s i, \backslash \text { theta_\{b\}\}\$ decreases }$ at increasing $\$$ theta_ $\{b\} \$$ as a function of the shape factor $\$ \backslash$ beta_ $\{\backslash$ theta_ $\{b\}\} \$ . \mid \backslash$

Biofilm dynamics are strongly readjusted when DOC is scarce $\left(\$ x i_{-}\{D O C\}<1 \$\right.$ ). On one hand, microorganisms are able to decompose EPS, using them as a carbon source to produce AC exclusively \citep[in agreement with][]\{DeSilva2000\}. The process is governed by the $\$ \backslash$ overline $\{\mathrm{EZ}\}$, which catalyze the reaction \citep\{Flemming2010\}, and $\$ x i_{-}\{E P S\}$. The latter term hampers the decomposition rates of EPS when their concentration is low compared to the value of \$EPS_\{K\}\$ (recall ( $\backslash$ ref $\{$ eqSM5\})). When EPS reuse occurs, the CUE drops because a lower percentage of carbon is assimilated (\$Y\$ is first applied in ( $\backslash$ ref $\{$ eqSM10\}) to produce EPS and then in ( $\backslash$ ref $\{$ eqSM9\}) to synthetize AC). On the other hand, some microorganisms switch in and out from the dormancy state (\ref\{eqSM11\}) according to nutrient availability and always fulfilling the restrictions imposed by population density defined by (\ref\{eqSM8\}). Such a 
physiological response occurs at a rate that is a function of $\$ \backslash t a u \_\{i\} \$, \$ \mid t a u \_\{a\} \$$, and \$xi_\{DOC $\} \$ . \mid 1$

Biomass losses are controlled by processes of decay, including the ageing effects (natural decay), the demands for maintenance and functioning (endogenous decay), and the reallocation of carbon (induced decay). Decay rates are defined proportional to the amount of biomass and the decay coefficients $\$ K \_\{D\}^{\wedge}\{A C\} \$, \$ K \_\{D\}^{\wedge}\{D C\} \$$, $\$ K \_\{D\}^{\wedge}\{E P S\} \$$ and $\$ K{ }_{-}\{D\}^{\wedge}\{E Z\} \$$, in which the subindices indicate the corresponding compartment. DC and EPS are the compartments least affected by decay $\left(\$ K \_\{D\}^{\wedge}\{A C\} / K \_\{D\}^{\wedge}\{D C\}>>1 \$, \quad \$ K \_\{D\}^{\wedge}\{A C\} / K \_\{D\}^{\wedge}\{E P S\}>>1 \$\right), \quad$ conferring large durability regardless of environmental conditions. The fraction \$\lambda_\{D\}\$ of decayed cells is assumed biodegradable and remains in the system becoming part of the EPS \citep[as in][]\{Laspidou2002b,Laspidou2004a\} and not POM Icitep[as in][]\{Riley2014\}. Such a simple mechanism allows producing EPS as a form of carbon reallocation \citep[e.g., in][]\{Kakumanu2013\}. Later on, these EPS can be used as a carbon source. As a consequence, the total consumption rate is defined by $\$ Y \$$ times the sum of DOC used and EPS decomposed.

Table 1. Nomenclature, parameter definition, and units of the model parameters.

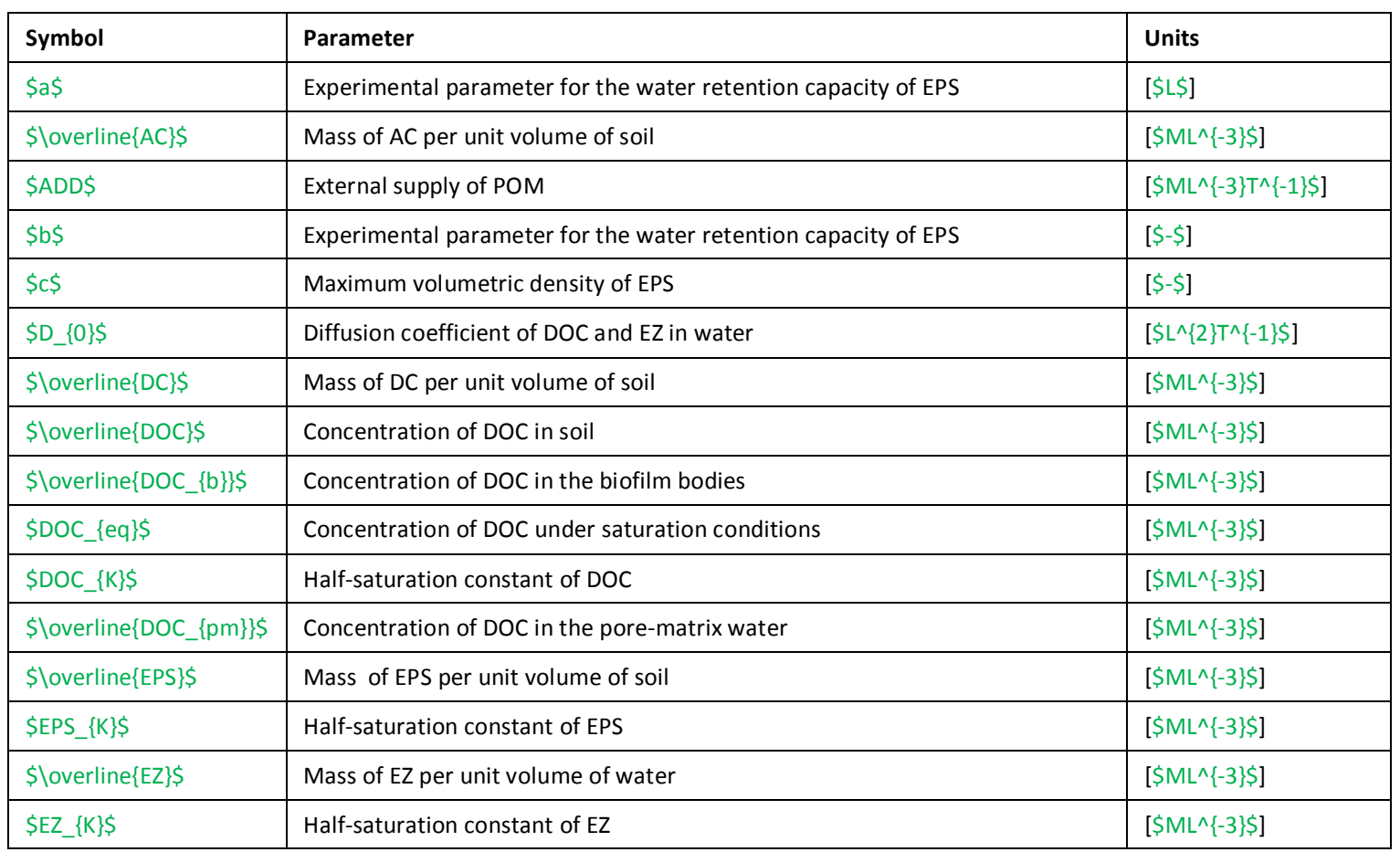




\begin{tabular}{|c|c|c|}
\hline$\$ K_{-}\{D\} \wedge\{A C\} \$$ & Decay rate coefficient for $\mathrm{AC}$ & {$\left[\$ T^{\wedge}\{-1\} \$\right]$} \\
\hline$\$ K_{-}\{D\} \wedge\{D C\} \$$ & Decay rate coefficient for $\mathrm{DC}$ & $\left.\left[\$ T^{\wedge}\{-1\} \$\right\}\right]$ \\
\hline$\$ K K_{i}\{D\} \wedge\{E P S\} \$$ & Decay rate coefficient for EPS & {$\left[\$ T^{\wedge}\{-1\} \$\right.$} \\
\hline$\$ K_{-}\{D\} \wedge\{E Z\} \$$ & Decay rate coefficient for EZ & {$\left[\$ T^{\wedge}\{-1\} \$\right]$} \\
\hline$\$ n \$$ & Experimental parameter for the SWRC of the biofilm-free soil & {$[\$-\$]$} \\
\hline \$loverline $\{\mathrm{POM}\} \$$ & Mass of POM per unit volume of soil & {$[\$ M L \wedge\{-3\} \$$} \\
\hline$\$ P O M \_\{K\} \$$ & Half-saturation constant of POM & {$[\$ M L \wedge\{-3\} \$]$} \\
\hline \$t\$ & Time variable & [\$\$T\$ \\
\hline \$t_\{c $\} \$$ & Characteristic time of the effects of the rain & [\$\$T\$ \\
\hline \$t_ $\{R\} \$$ & Time of the last rainfall event & [\$\$T\$ \\
\hline$\$ v_{-}\{w\} \$$ & Averaged flow velocity of water & {$[\$ L T \wedge\{-1\} \$$} \\
\hline \$Y\$ & Yield coefficient of respiration & {$[\$-\$]$} \\
\hline$\$ z \$$ & Coordinate parallel to the water flow direction & [S\$L\$ \\
\hline \$lalpha\$ & Van Genuchten's parameter for the SWRC of the biofilm-free soil & {$\left[\$ L^{\wedge}\{-1\} \$\right]$} \\
\hline \$beta_\{P\}\$ & Shape factor that regulates the curvature of $\$ \backslash x i \_\{\mid p s i, P\} \$$ & {$[\$-\$]$} \\
\hline \$beta_\{\theta_\{b\}\}\$ & Shape factor that regulates the curvature of $\$ \backslash x i_{-}\{\mid p s i, \backslash$ theta_ $\{b\}\} \$$ & {$[\$-\$$} \\
\hline$\$ \backslash$ Deltat_\{R\}\$ & Return period of rainfall events & [\$\$\$ \\
\hline \$gamma\$ & Shape parameter for the solute diffusion coefficient & {$[\$-\$]$} \\
\hline \$Gamma\$ & Mass transfer function & {$\left[\$ M L \wedge\{-3\} T^{\wedge}\{-1\} \$\right]$} \\
\hline \$|theta_\{b\}\$ & Volume of water in biofilm bodies & {$[\$-\$$} \\
\hline \$theta_\{pm\}\$ & Volume of pore-matrix water & {$[\$-\$]$} \\
\hline \$|theta_\{r\}\$ & Residual water content in the soil & {$[\$-\$]$} \\
\hline \$|theta_\{s\}\$ & Saturated water content in the soil & [\$-\$] \\
\hline$\$ \backslash$ lambda_\{d $\} \$$ & Decayed mass reallocation ratio & [\$-\$] \\
\hline$\$ \backslash$ lambda_\{EPS\}^\{*\}\$ & Effective coefficient of carbon allocation diverted towards EPS & {$[\$-\$]$} \\
\hline \$Vlambda_\{EPS\}\$ & Maximum coefficient of carbon allocation diverted towards EPS & {$[\$-\$]$} \\
\hline$\$ \backslash$ lambda_ $\{E Z\}^{\wedge}\left\{{ }^{*}\right\} \$$ & Effective coefficient of carbon allocation diverted towards EZ & {$[\$-\$]$} \\
\hline \$Vlambda_\{EZ\}\$ & Maximum coefficient of carbon allocation diverted towards EZ & [\$-\$] \\
\hline \$N\$ & Experimental parameter denoting the channeled architecture of biofilms & [\$-\$] \\
\hline \$mu_\{c\}\$ & Maximum specific consumption rate & {$\left[\$ T^{\wedge}\{-1\} \$\right]$} \\
\hline \$Imu_\{EPS\}\$ & Maximum specific decomposition rate of EPS & {$\left[\$ T^{\wedge}\{-1\} \$\right.$} \\
\hline \$Imu_\{POM $\} \$$ & Maximum specific decomposition rate of POM & {$\left[\$ T^{\wedge}\{-1\} \$\right.$} \\
\hline \$xi_\{DOC $\} \$$ & $\begin{array}{l}\text { Environmental coefficient based on the concentration of DOC in the biofilm } \\
\text { phase }\end{array}$ & {$[\$-\$$} \\
\hline \$ $\mid x i \_\{E P S\} \$$ & Environmental coefficient based on the concentration of EPS & {$[\$-\$]$} \\
\hline \$\} | x i \_ \{ E Z \} \$ $&{\text { Environmental coefficient based on the concentration of EZ }} &{\text { [\$-\$] }} \\
{\hline \text { \$XI_\{POM\}\$ }} &{\text { Environmental coefficient based on the concentration of POM }} &{\text { 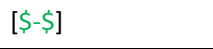 }} \\
{\hline \$ \mid x i \_\{\mid t a u\} \$} &{\text { Solute diffusion coefficient }} &{[\$-\$]} \\
{\hline \$ \mid x i \_\{\mid c h i\} \$} &{\begin{array}{l}\text { Environmental coefficient based on the concentration of the generic variable } \\
\text { \$\overline\{chi\}\$ }\end{array}} &{[\$-\$} \\
{\hline \text { \$\} | x i \_ \{ \backslash p s i , P \} \$ } &{\text { Quorum sensing coefficient based on the cell population }} &{\text { [\$-\$] }} \\
{\hline \$ \mid x i \_\{\backslash p s i, \mid \text { theta_\{b\}\}\$ }} &{\text { Quorum sensing coefficient based on } \$ \mid \text { theta_ }\{b\} \$} &{[\$-\$} \\
{\hline \text { \$rho_\{c\}\$ }} &{\text { Density of the microbial cells }} &{[\$ M L \wedge\{-3\} \$} \\
{\hline \text { \$rho_ }\{w\} \$} &{\text { Density of water }} &{[\$ M L \wedge\{-3\} \$} \\
{\hline \text { \$tau_\{a\}\$ }} &{\text { Maximum rate of cell reactivation }} &{\left[\$ T^{\wedge}\{-1\} \$\right.} \\
{\hline \text { \$tau_\{i\}\$ }} &{\text { Maximum rate of cell inactivation }} &{\text { 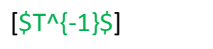 }} \\
{\hline \text { \$\phi_\{ef\}\$ }} &{\text { Effective porosity of the soil }} &{[\$-\$]} \\
\hline\end{array}$
\end{tabular}




\begin{tabular}{|c|c|c|}
\hline \$phi_\{M $\} \$$ & Maximum effective pore volume susceptible to be occupied & [\$-\$] \\
\hline 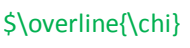 & Mass of the generic variable $\$ \backslash$ loverline $\{\mid c h i\}$ per unit volume of water or soil & {$[\$ M L \wedge\{-3\}]$} \\
\hline \$\Chi_\{K\}\$ & Half-saturation constant of the generic variable $\$ \backslash$ overline $\{\backslash c h i\}$ & {$[\$ M L \wedge\{-3\}]$} \\
\hline \$ \psi\$ & Matric suction & [\$\$L\$ \\
\hline \$\psi_\{0\}\$ & Matric suction in the natural state of the soil & [\$\$ \\
\hline \$Omega\$ & Shape parameter for the solute diffusion coefficient & {$[\$-\$$} \\
\hline
\end{tabular}

\section{|subsection\{Numerical Simulations Set-Up\}}

The coupled system of equations presented in (\ref\{eqSM1\}-\ref\{eqSM15\}) was solved numerically using an implicit Crank-Nicolson scheme. Concentrations of the studied compartments were updated every time step. Such steps were dynamically chosen according to convergence criteria, and to avoid numerical instabilities. The resulting time steps were in the order of seconds-minutes. Each simulation was performed until quasi-steady-state conditions were achieved; i.e., when no relative changes in the biomass of all compartments were observed (for constant environmental conditions), or temporal patterns reached a stable cycle (for cyclic environmental conditions). A minimum time of simulation was set to $\$ 365 d \$$, which was assumed the behavior at long-term. $\ 1$

All simulations were performed in a porous medium of $\$ 1 \mathrm{~cm}^{\wedge}\{3\} \$$ volume, albeit the results can be extrapolated to larger scales by combining multiple volumes. Different sets of simulations were carried out to conduct a sensitivity analysis in which either one parameter or combinations of two parameters were allowed to vary. Most of these parameters were set based on the literature, while others (when no information was available) were constrained to a reasonable range or value (Table 2). Due to the high sensitivity and little knowledge of the value of the parameters under each scenario, simulations were employed then as an explorative rather than predictive tool to disentangle qualitative patterns and the driving forces governing microbial dynamics under different situations of stress.

Simulations were conducted in synthetic scenarios in order to explore the carbon dynamics in shallow soils. Supplies feeding the system were adapted to simulation needs, differentiating between two main scenarios. The first scenario assumed that 
the microbial community was fed by the decomposition of POM accumulated in the upper soil profile, i.e., DOC was not directly supplied. To focus on the effects of DOC limitation, \$ \overline $\{\mathrm{POM}\}$ was assumed at steady-state and set to a constant value,

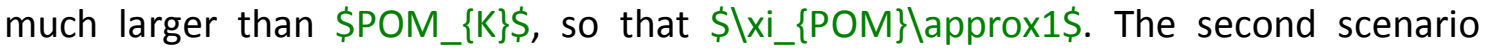
considered artificial sudden changes in the availability of DOC. Therefore, the mass balance for POM (in (\ref\{eqSM3\})) became redundant for the scenarios studied. Additionally, the mass transfer rate between the two DOC compartments is assumed large enough to maintain equilibrium, and therefore \$loverline $\left\{D O C \_\{b\}\right\}$ and \$ \overline $\left\{D O C \_\{p m\}\right\}$ are equal to the concentration \$\overline $\{D O C\} \$$ in the now undistinguished dissolved pool. The inflow/outflow diffusion fluxes were considered balanced and could be neglected. Additional details are provided later in each casestudy section.

To explore the wide range of saturations found in natural environments, simulations were performed on soils under synthetic extreme scenarios consisting in: full saturation, moderately dry conditions, or intermittent rainfall events. Such conditions were specified by the soil suction status and the availability of water. The saturated scheme assumed that $\$ \backslash p s i=0 \$$ and therefore the volumetric water content was maximum and equal to \$|theta_\{s\}\$. The dry scenario assumed conditions of water limitation (with no additional water supplied during simulations) where $\$$ theta_\{b\}+\theta_\{pm\}\$ was determined by the equilibrium in (\ref $\{$ eqSM1\}) and (\ref\{eqSM2\}) at a constant $\$$ psi\$ of $\$ 50 \mathrm{~cm} \$$ of water, regardless of microbial dynamics. When biofilm proliferated, the equilibrium was lost and \$phi_\{M\}\$ was set to the value of $\$$ theta_ $\{b\}+\backslash$ theta_ $\{\mathrm{pm}\} \$$ at $\$ t=0 \$$. Finally, the scenarios of intermittent rainfall events started from a dry scenario that included periodic sudden raises of the water content ( $\$ p s i=0 \$$, representing the rainfall event) with a return period $\$ \backslash D e l t a t \_\{R\} \$$. Water inputs were assumed at zero concentration of DOC and EZ to represent the conditions in a very shallow soil. The composition of the input water was still similar to that of rainwater, entailing a potential dilution of these components. After the event, soils underwent a transient period of pressure redistribution, gradually readapting their saturation to the natural suction state (\$\} \backslash \text { psi_\{0\}=50cm\$). Water drainage flushed out a fraction of the dissolved } 
components, preserving their concentrations. The effect of matric suction was not studied as an independent stress, but only as a driver of the water content (assuming (\ref\{eqSM1\}) and (\ref\{eqSM2\}) at equilibrium). The changes in suction were modeled as

$$
\psi(t)=\psi_{0}-\psi_{0} \exp \left(-\frac{t-t_{R}}{t_{c}}\right)
$$

where $\$ t \_\{\} \$$ is the time of the last rainfall event and $\$ t\{f c\} \$$ is the characteristic time of the effects of the rain on $\$ \backslash p s i \$$. The time of the first episode was set to $\$ \backslash D e l t a$ $\mathrm{t} \_\{\mathrm{R}\} / 2 \$$.

\section{|section\{Results and Discussion\}}

We start by evaluating the effect of soil texture and biofilm characteristics, i.e., retention properties and pore-scale morphology. We then continue by examining the deployment of survival mechanisms responding to stresses characterized by the synthesis of EPS, their reuse as a source of $C$, the inactivation/reactivation of cells, and the combined effect of dormancy and EPS production.

\section{Isubsection\{The Effect of Soil Type and Biofilm Properties\}}

The soil pore-size distribution strongly determines the availability of elements essential for the proliferation of biofilms. First, the water saturation, which is a key factor in microbial habitats, is itself a function of the size, the shape and the interconnection of pores \citep\{Alaoui2011\}. This relationship determines the SWRC (see Supplementary material Figure \ref\{figSM1S\}), which typically depicts lower saturations at a given matric suction in coarse grain soils because large pores can be easily drained. As an example, using the SWRCs reported by \citet\{Carsel1988\}, sands exhibit an $\$ 86 \backslash \% \$$ reduction of their water content at a suction of $\$ 50 \mathrm{~cm} \$$. For sandy clays, this value is about $\$ 12 \backslash \% \$$, and it does not exceed $\$ 40 \backslash \% \$$ at $\$ 1000 \mathrm{~cm}$ suction. Second, DOC availability not only depends on water saturation but also on diffusion capacity. Substrate supply turns into a prominent limiting factor when water content is low since diffusive and conductive transport becomes small \citep\{Manzoni2012a\}. Even 
though the water holding capacity of fine-textured soils is frequently larger than that of coarse-textured soils, the increased tortuosity of the diffusion paths in their thin pores (lower $\$ \backslash x i_{1}\{\backslash \operatorname{tau}\} \$$ ) reduces the capacity of solutes to reach the microbial cells. According to (\ref\{eqSM4\}), such diffusion limitation is determined by the parameters \$Omega\$, \$gamma\$, and \$theta_\{s\}\$, which are soil-type dependent. It is worth noting here that the model does not account for increased carbon losses that may occur in soils of low tortuosity (with potentially higher advection-diffusion fluxes), and for reduced microbial growth caused by oxygen limitation that frequently occurs in highly saturated environments \citep[see][for details]\{Skopp1990\}.\\

These hydraulic properties are not static, but depend on the presence of biomass, which lead to direct (\$theta_\{b\}\$) and indirect (\$theta_\{pm\}\$) increases in the overall retention of water for a given suction.. In Figure \ref\{figSM1S\}, the retention properties of biofilms are illustrated by comparing the effect determined by an EPS of a very low capacity ( $\$ a \$$ assumed equal to $\$ 1 \mathrm{~cm} \$$ ), with that of pure xanthan (with better retention properties, $\$ a=105.76 \mathrm{~cm} \$)$. The first scenario requires many times the mass of xanthan to obtain an analogous change in the SWRC. Therefore, as \$b\$ and $\$ c \$$ are here assumed independent of the type of EPS, the overall change in saturation is governed by the product $\$ a \$ x \$ \backslash$ \overline\{EPS\}\$ (recall (\ref\{eqSM1\})). Additionally, the more intricate is the architecture of the biofilm at the pore-scale (denoted by $\$ N \$$ ), the stronger the transformation of the pore-matrix and the higher is the total saturation increase. According to the previous statements and the model equations, an increase of water saturation implies a potential increase in the rates and effectiveness of carbon consumption by microbial cells. The influence of EPS on microbial dynamics is discussed in more detail in Section 3.2, but a simple analysis of the results shown in Iref\{figSM1S\} already suggests that the greater the impact of biofilm on the SWRC is, the more benefits are potentially achieved by cells. This point supports the speculation of Icitet\{Brangari2017\} and Icitet\{Lawrence1991\},who suggested that the intricate architecture of biofilms enhances the nutrient uptake. \I 

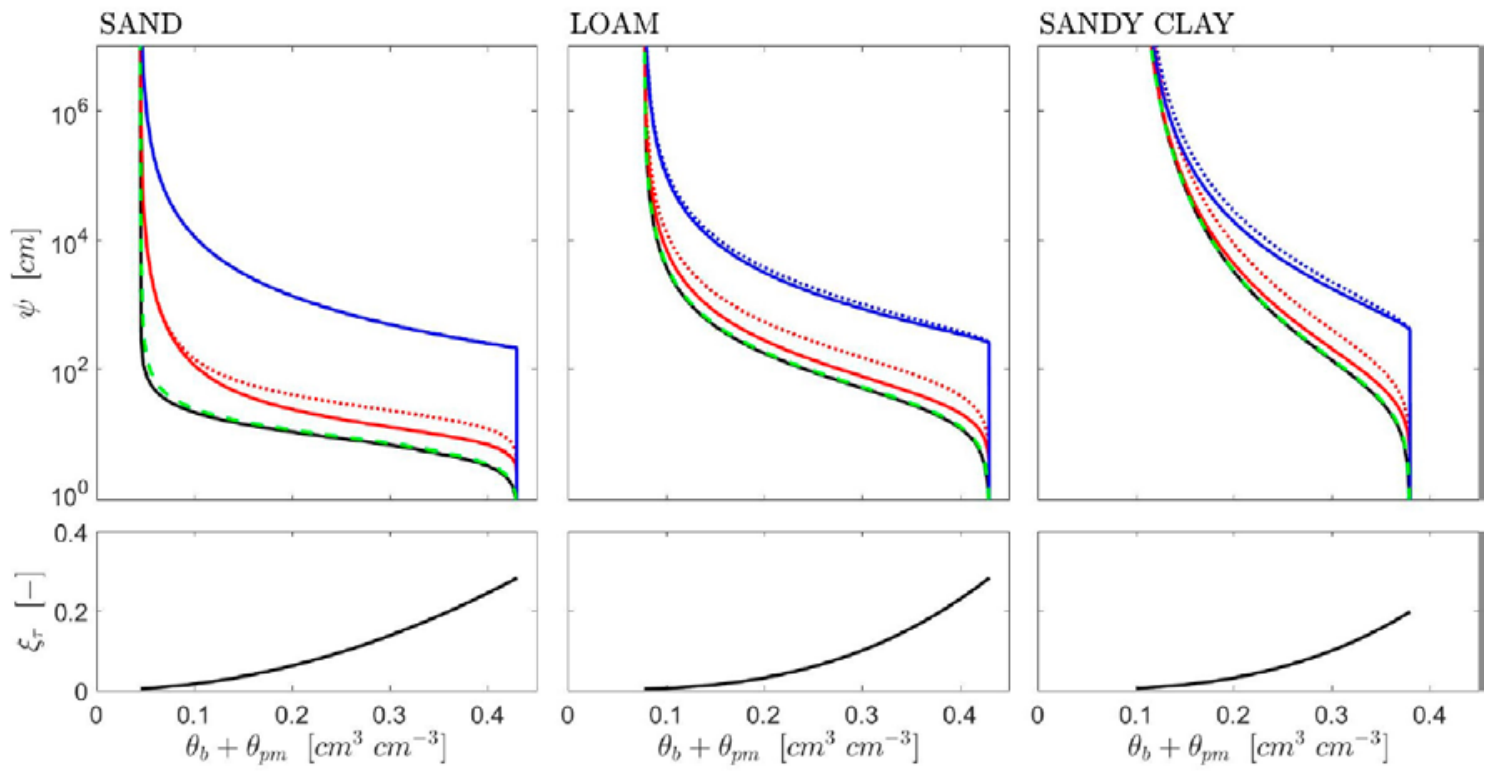

$\square \overline{E P S}=0 \quad|N=1| a=105.76$
$-\overline{E P S}=0.005|N=1| a=105.76$
$\ldots \ldots . \overline{E P S}=0.005|N=5| a=105.76$

$-\overline{E P S}=0.05|N=1| a=105.76$
$\cdots \cdots \overline{E P S}=0.05|N=5| a=105.76$
$--\overline{E P S}=0.05|N=1| a=1$

\section{\subsection\{The Effect of EPS on Microbial Dynamics\}}

\section{\subsubsection\{Water Availability and Hydric Stress\}}

In this section, we analyze the impact of EPS accumulation under variable soil moisture on the proliferation of biofilms. To better identify the effects of hydric stress and the role of EPS, we simulate the effect of having or not having colonies embedded in a polymeric matrix (\$0.015\$ or $\$ 0 \$$ g of EPS $\$ \mathrm{~cm}^{\wedge}\{-3\} \$$ ), indicated by dotted and solid curves in Figure \ref\{figSM2\}, respectively. The retention capacity of EPS was taken from that of pure xanthan to emphasize its impact on soils. The production of additional EPS, their decay, and the switch into dormancy were all deactivated in these simulations by setting $\$ \backslash$ lambda_ $\{E P S\}=0 \$, \$ K \_\{D\}^{\wedge}\{E P S\}=0 \$, \$ \backslash l a m b d a \_\{d\}=0 \$$, and $\$ \backslash$ tau_ $\{i\}=0 \$$. In this way, the EPS pool was treated as a static compartment, allowing us to isolate its effects. Results compare the proliferation of cells in a soil under moderately dry and fully saturated conditions (in panel A), and under instantaneous rainfall events that are followed by drainage periods of $\$ 5 \$$ and $\$ 10 \$$ days in duration (in panel B). \I

In moist conditions (absence of major stresses and of dilution effects), microorganisms grew fast, efficiently, and reached large active biomass values (blue curves in IrefffigSM2\}A). Large POM decomposition rates permitted high respiration rates and 
activity (recall that $0 \$\{\{2\} \$$ is not considered a limiting factor). As shown by the overlapping solid and dashed blue curves in Figure \ref\{figSM2\}A, the presence of EPS did not have an apparent role under such circumstances. In contrast, in rather dry soils, the growth of microorganisms was limited by the availability of DOC (due to low \$ $\backslash x i \_\{\text {tau\}\$), which hampered the synthesis of new biomass (green curves in }$ Jref\{figSM2\}A). The presence of EPS (dotted curves) in such dry scenarios increased water saturation, contributing to the improvement of microbial fitness compared to cases without EPS (solid curves). \I

According to \citet\{Dutta2015\} and \citet\{Fierer2002\}, the periodicity and intensity of the stress episodes control the proliferation of microbial colonies. Along this line, results in Figure \refffigSM2\}B show a significant increase in the respiration rates during each rainfall event \citep[known as Birch effect;][]\{Birch1958\}. The enhancement of the decomposition rates by the sudden increase in the saturation was counterbalanced by the dilution of EZ and DOC. Thereafter, soils transiently readapted their water content to \$psi_\{0\}\$ triggering losses of dissolved components and EZ by leaching. Those colonies embedded in EPS attained the largest cell populations; effect that is more pronounced under scenarios with higher rainfall frequency. A detailed analysis of the results showed that EPS caused mitigation in both water and carbon stresses by: i) emulating the conditions of colonies growing at a smaller hydric stress that remain saturated for a wider range of suctions \citep\{Or2007a\}; ii) acting as a reservoir that retains $\mathrm{DOC}$ and $\mathrm{EZ}$, which mitigates the effects of dilution and drainage \citep\{Or2007b\}; and iii) increasing DOC supply during dry periods \citep\{Chenu1996\}. These points may indicate feasible mechanisms by which EPS allowed microorganisms to benefit from rainfall events that otherwise would inhibit biofilm proliferation. This result provides also possible insights on the drivers of respiration pulses at rewetting Icitep\{Birch1958,Lado2014,Meisner2013\}. For a pulse to occur, DOC and EZ must be retained in the system, which is more feasible with a well-developed EPS matrix. This link between respiration pulses and EPS had not been previously explored, and emerges in the dynamics only when the complexity of microbial-soil pore space is described. 


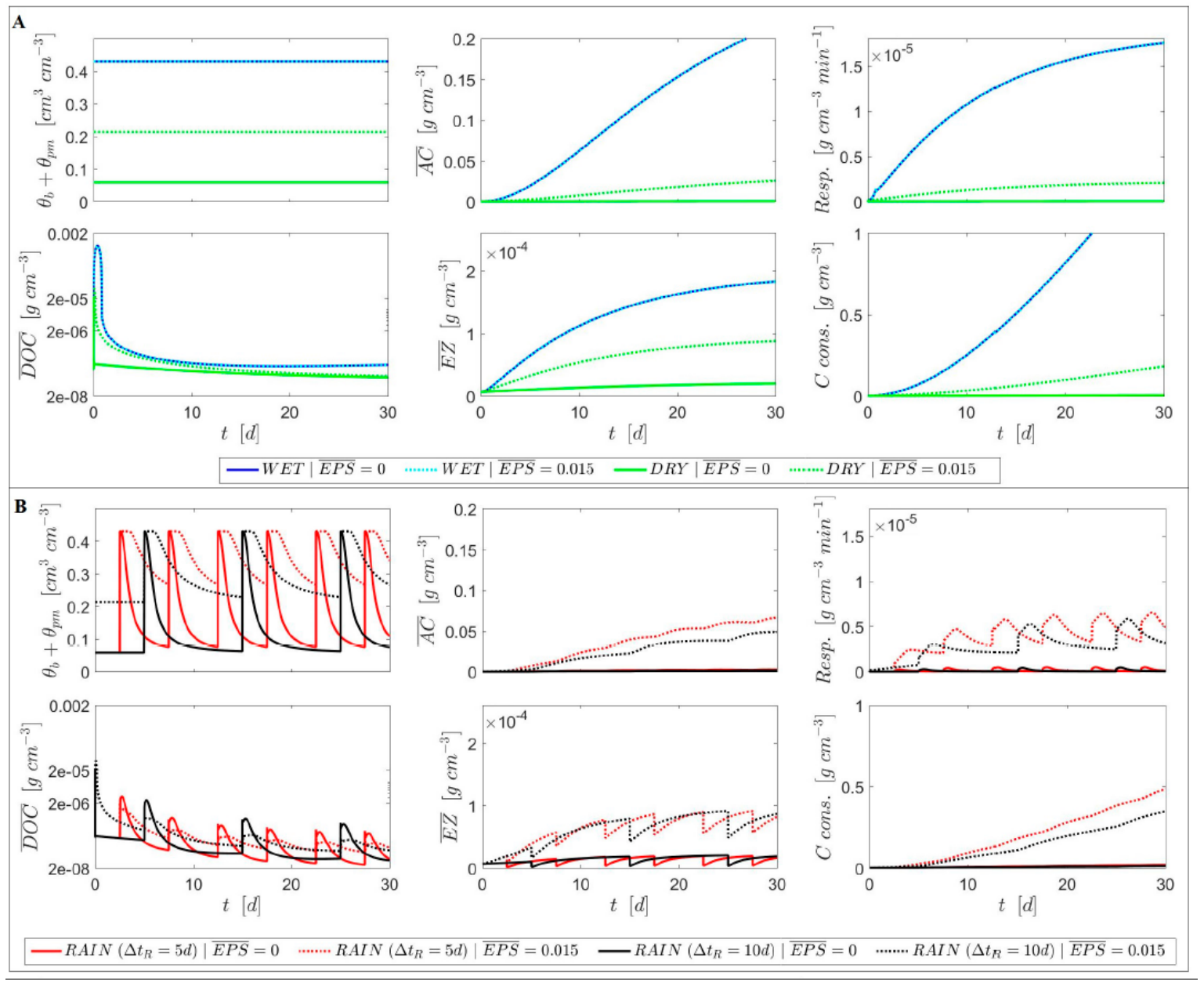

Icaption\{Impact of water availability on microbial dynamics. Simulations were performed for a soil under A) moderately dry conditions (green), fully saturated (blue), and B) under instantaneous rainfall events followed by drainage periods $\$ \backslash D e l t a t \_\{R\} \$$ of $\$ 5 \$$ days (red) and $\$ 10 \$$ days (black). The colony of microorganisms modeled was assumed to be either embedded (\$\overline\{EPS\}=0.015 $\mathrm{g} \$ \$ \mathrm{~cm} \wedge\{-3\} \$$, dotted lines) or not (\$overline $\{E P S\}=0 \$$, solid lines) in a polymeric matrix. Multiple plots show the dynamics of the total water content \$|theta_\{b\}+\theta_\{pm\}\$, the active cell population \loverline $\{\mathrm{AC}\} \$$, the respiration rate (indicated by Resp.), the dissolved organic carbon concentration \$loverline $\{\mathrm{DOC}\} \$$, the enzyme density $\$ \backslash$ overline $\{\mathrm{EZ}\} \$$, and the cumulative amount of carbon consumed (C cons.).\}

\section{Isubsubsection\{Production of EPS\}}

Here, the simulations are extended by introducing the dynamics of EPS. We recall that in Section 3.2.1 the EPS concentration was set to a constant parameter. The hypothesis being tested here was that EPS should provide an advantage to the organisms embedded, even though some resources are diverted from cell growth towards their production. This effect was studied by looking at the proliferation of organisms 
characterized by increasing the potential allocation of carbon to the production of EPS

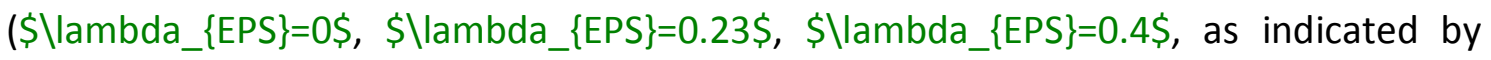
different colors in Figure \ref\{figSM3\}). Unlike previous simulations, EPS production (determined by environmental stresses represented by (\ref\{eqSM12\})) and decay (stress independent) were activated so that EPS dynamically interacted with other compartments. The columns of Figures \ref\{figSM3\} and \refffigSM4\} show a selection of some relevant simulations that illustrate biofilm dynamics under different saturation levels (two different rainfall frequencies and full saturation). $\ \backslash$

Under saturated conditions, since the production of large amounts of EPS was unfruitful (Figure \refffigSM3\}, right), the resources diverted to EPS were downregulated. $\$ \backslash \mid a m b d a \wedge\{*\}\{\{E P S\}$ tended to zero and $\$ \backslash$ overline $\{E P S\} \$$ reached a pseudo-steady state. This phenomenon might explain why environments under small water stress tend to exhibit low EPS to biomass ratios Icitep[e.g., in][]\{Rubol2014,Vandevivere1992b\}. As a consequence of regulation, the dynamics obtained were not sensitive to \$\lambda_\{EPS\}\$. When such a mechanism was disabled (by assuming $\$ \mid x i \_\{\backslash p s i, \backslash \text { theta_\{b\}\}=1\$, not shown), EPS were continuously }$ synthetized at the expenses of population growth. \I 

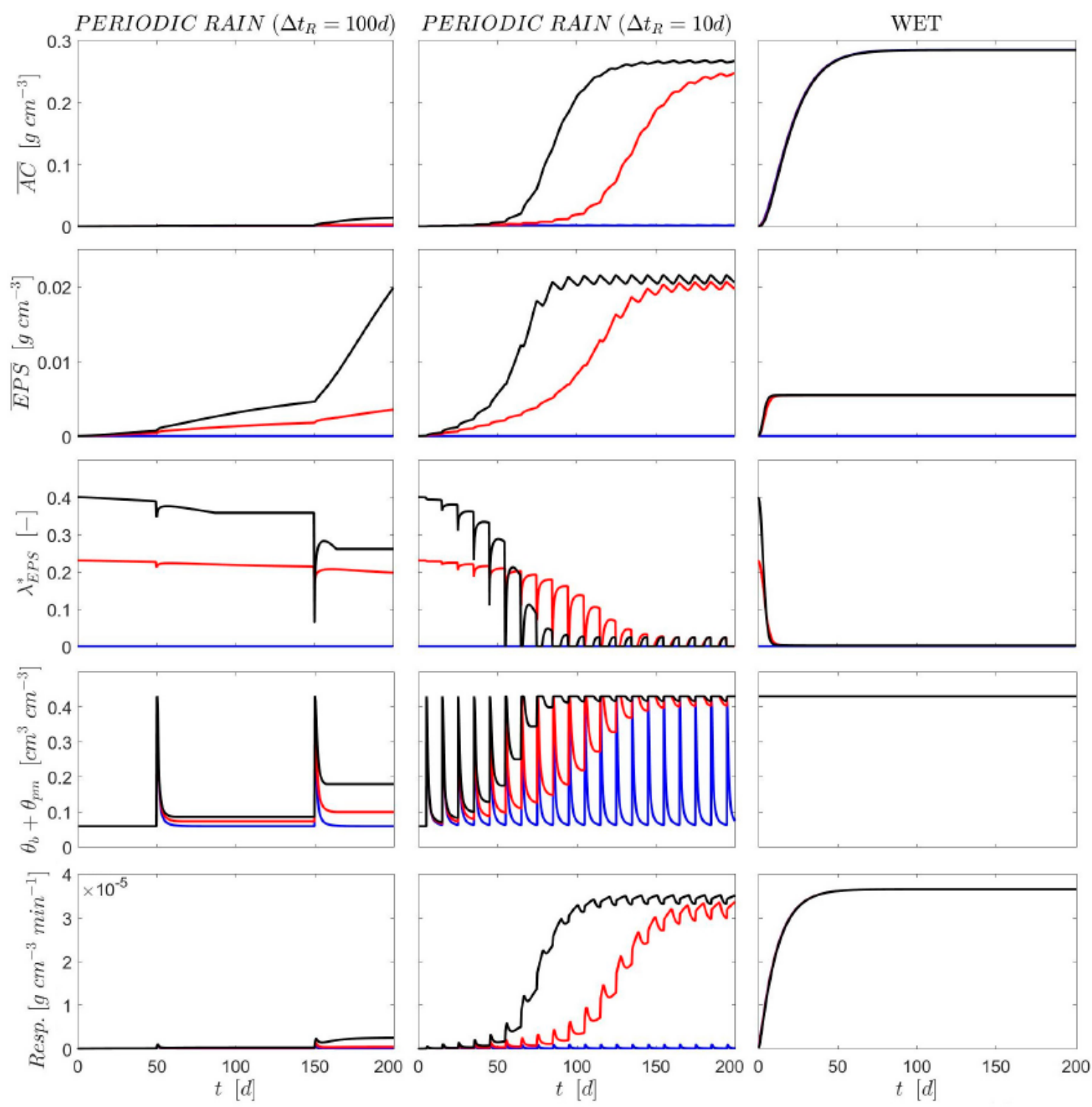

$-\lambda_{E P S}=0-\lambda_{E P S}=0.23-\lambda_{E P S}=0.4$

Icaption\{Simulation of biofilm proliferation under permanent wet conditions (plots in the right column), and environments affected by rainfall events with a return period of $\$ 100 \$$ (left column) and $\$ 10 \$$ days (center column). From top to bottom: active microbial biomass (\$loverline\{AC\}\$), amount of EPS (\$loverline\{EPS\}\$), effective coefficient of carbon allocation to synthetize new EPS $\left(\$ \backslash\right.$ lambda_ $\left.\{\mathrm{EPS}\}^{\wedge}\left\{{ }^{*}\right\} \$\right)$, total water content $(\$ \backslash$ theta_$\{\mathrm{b}\}+\backslash$ theta_ $\{\mathrm{pm}\} \$)$, and respiration rate (Resp.). The maximum coefficient of carbon allocation diverted towards EPS \$\lambda_\{EPS\} values are $\$ 0 \$$ (blue), \$0.23\$ (red), and \$0.4\$ (black).\}

Dynamics under variable soil moisture are illustrated in Figure \ref\{figSM3\} (left and center columns) by showing the results under periodic rainfall events with a return period of $\$ 100 \$$ (left) and $\$ 10 \$$ days (center). Under such conditions, the large allocation of carbon to EPS resulted in significant improvements in microbial fitness (i.e., biomass). Yet, results show that microorganisms required moderately long 
periods to adapt the biofilm (and the soil habitat) to their needs. With time, each microbial system tended to reach high active biomass values as long as the production of EPS was larger than their decay. Note the black and red curves in the top panel of Figure \ref\{figSM3\} that microbial bloom occurred when biofilm helped preserving (proper) conditions after a rainfall event. Moreover, the more frequent were the rain spells, the faster the steady-state conditions were achieved, thanks to the higher mean soil moisture. On the other hand, if dry periods are sufficiently dry and intense, results (not shown) agreed with those obtained by Icitet\{Chang2003,Roberson1992,Sandhya2015\}, who demonstrated that colonies under desiccation may exhibit relatively high \$loverline\{EPS\}, reaching values up to $\$ 75 \$-\$ 85 \backslash \%$ of the total biomass. When there is no water available, such a large accumulation of EPS did not improve the hydration status and nutrient uptake. As a consequence, EPS investments were unfavorable. In some cases, particularly when \$Vlambda_\{EPS\}\$ was low, the simulation time required to reach equilibrium exceeded $\$ 5000 \$$ days. Figure \refffigSM4\} shows the averaged biomass after $\$ 1 \$$ year of simulation. Results suggest that for EPS to be effective, larger investments (\$\lambda_\{EPS\}\$) are required under progressively more stressful conditions. \I

According to the observations in \citet\{Romani2008\}, during the early stage of biofilm development, an increase in the relative proportion of EPS was observed (in Figure \ref\{figSM3\}, but not appreciable in the current y-axis scale). For the studied set of parameters, the presence of a small amount of EPS did not compensate for the resources currently diverted to their production and \$ \overline $\{A C\} \$$ remained low compared to the non-producer strain. However, when \$loverline\{EPS\}\$ reached higher values, compensatory mechanisms started to work, increasing the respiration and the synthesis of new biomass (on average, but particularly during activity peaks, Figure \ref\{figSM3\}). As a result, highly bio-amended soils remained almost fully saturated even at high matric suctions, also counteracting the adverse diluting effects of rainfall events. In those scenarios with stronger water stress, larger amounts of EPS were accumulated, which facilitated conditions recovery after rewetting \citep[e.g.,][]\{Dutta2015\} and sustained a higher \$loverline\{AC\}\$ for larger \$Ylambda_\{EPS\}\$. This fitness improvement resulted from the dynamic synthesis of 
EPS in response to the intensity of the water stress. Production of EPS remained low during dry spells (\$\lambda^\{*\}_\{EPS\}<\lambda_\{EPS\}\$), but decreased or even stopped (\$\|ambda^\{*\}_\{EPS\}\rightarrow0\$) during rainfall events. Effective downregulation of EPS synthesis occurred when EPS could not provide further benefits in saturation (denoted by $\$ \mid x i \_\{\mid p h i, \backslash \text { theta_\{b\}\}), allowing organisms to divert }$ resources to AC. If the downregulation mechanism had been disabled, the synthesis of unnecessary matrix would have negatively affected microbial fitness. In that case, Figure \refffigSM4\} would have shown a bell-shape relation between \$loverline $\{A C\} \$$ and \$lambda_\{EPS\}\$. II

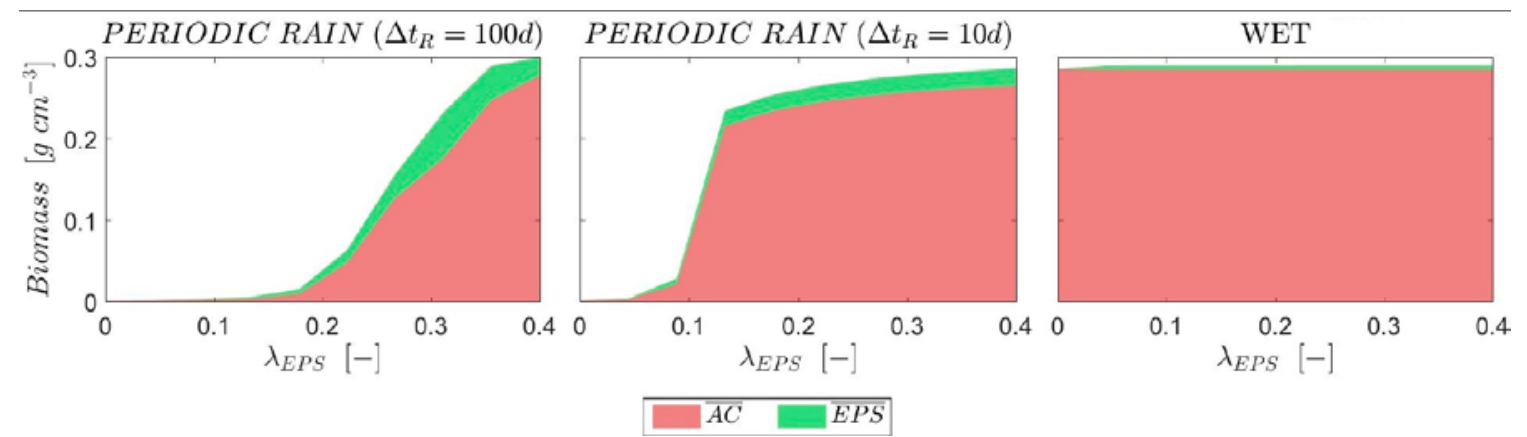

Icaption\{Partitioning of the biofilm compartments in long-term simulations (dry-wet cycle-averaged values after $\$ 1 \$$ year of simulation) assuming values of the maximum coefficient of carbon allocation diverted towards EPS (\$Vlambda_\{EPS\}) ranging from $\$ 0 \$$ to $\$ 0.4 \$$, and the maximum rate of cell inactivation \$ $\backslash$ tau_ $\{i\}=0 \$$ (dormancy was not allowed). Simulations were performed under periodic rainfall events with $\$ \backslash$ Delta $t \_\{R\}=100 \$$ days (left), $\$ 10 \$$ days (center), and permanently wet conditions (right).\}

As an alternative to the time series representation shown in Figure \ref\{figSM4\}, the relations between microbial metabolism and environmental conditions can be represented in the phase space, by showing respiration rate as a function of moisture (Supplementary material Figure \ref\{figSM2S\}). The model simulations showed that respiration rates decreased during soil drying, following a pattern that has often been observed \citep[see][for a review]\{Manzoni2012a\}. Upon rewetting, respiration rate increased with a short delay, partly due to DOC dilution effects, causing counterclockwise hysteretic loops \citep[as reported elsewhere, see][]\{Zhang2015\}. Therefore, the maximum respiration rate was achieved only when soil moisture had already started to decrease. 


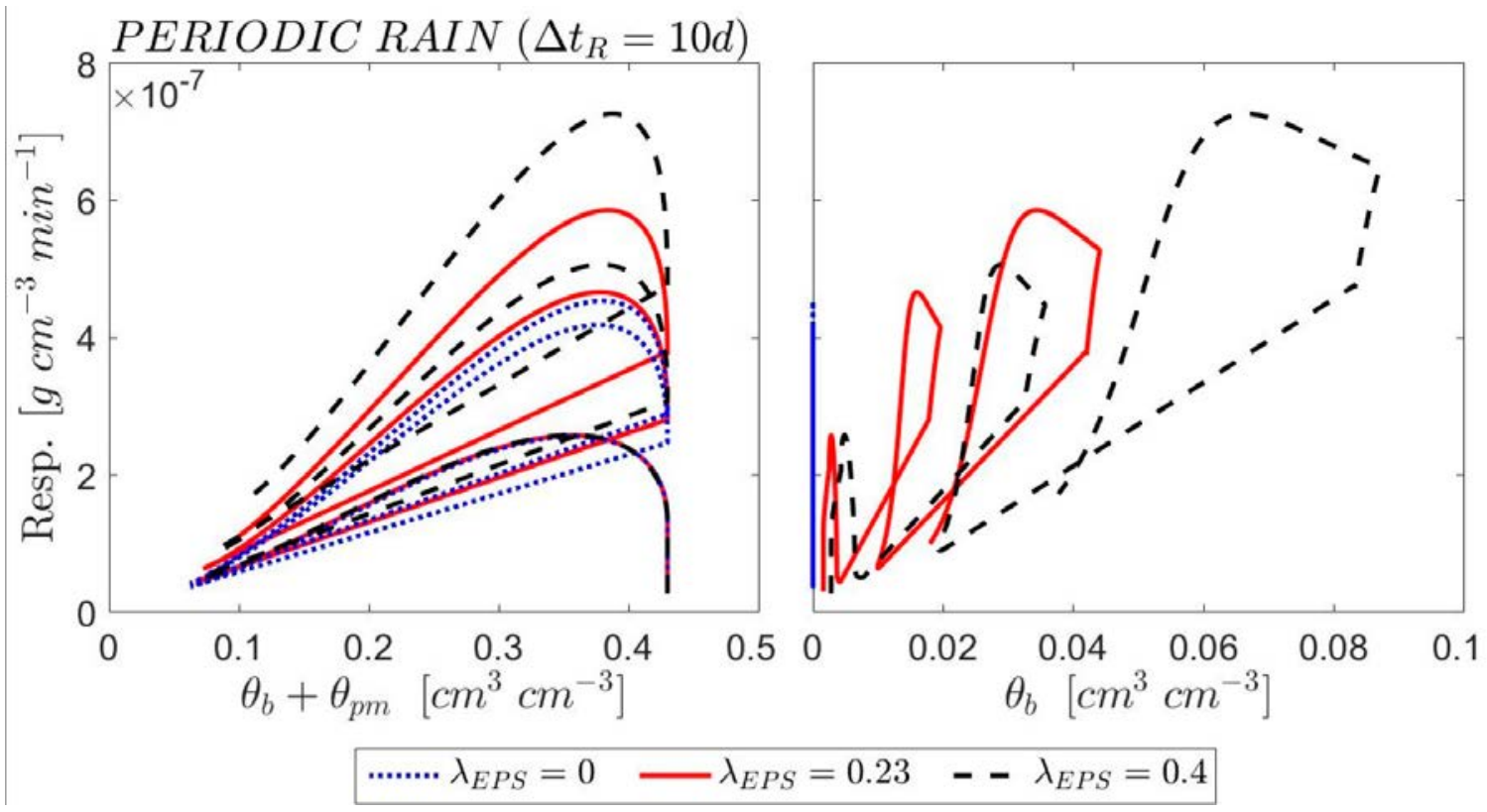

\section{\subsubsection\{Reuse of EPS\}}

The potential benefits of storing carbon in EPS for late use were tested by comparing EPS-producer microorganisms (\$\lambda_\{EPS $\}=0.23 \$$ ) with different capacity to reuse the carbon previously invested in EPS, assuming \$ $\backslash$ mu_\{EPS $\} \$$ equal to $\$ 1 \$ x \$ 10^{\wedge}\{-$ $3\} \min \wedge\{-1\} \$$ (reuse) or $\$ 0 \$$ (no reuse). Two different EPS water retention capacities were also studied comparing $\$ a=105.76 \$$ and $\$ a=1 \mathrm{~cm} \$$. Some relevant simulations obtained for these four combinations of \$Vlambda_\{EPS\}\$ and \$a\$ are shown in Figure \ref\{figSM5\}, for a soil affected by periodic rainfall events with $\backslash$ Delta $t \_\{R\}=10 \$$ days. $\ 1$

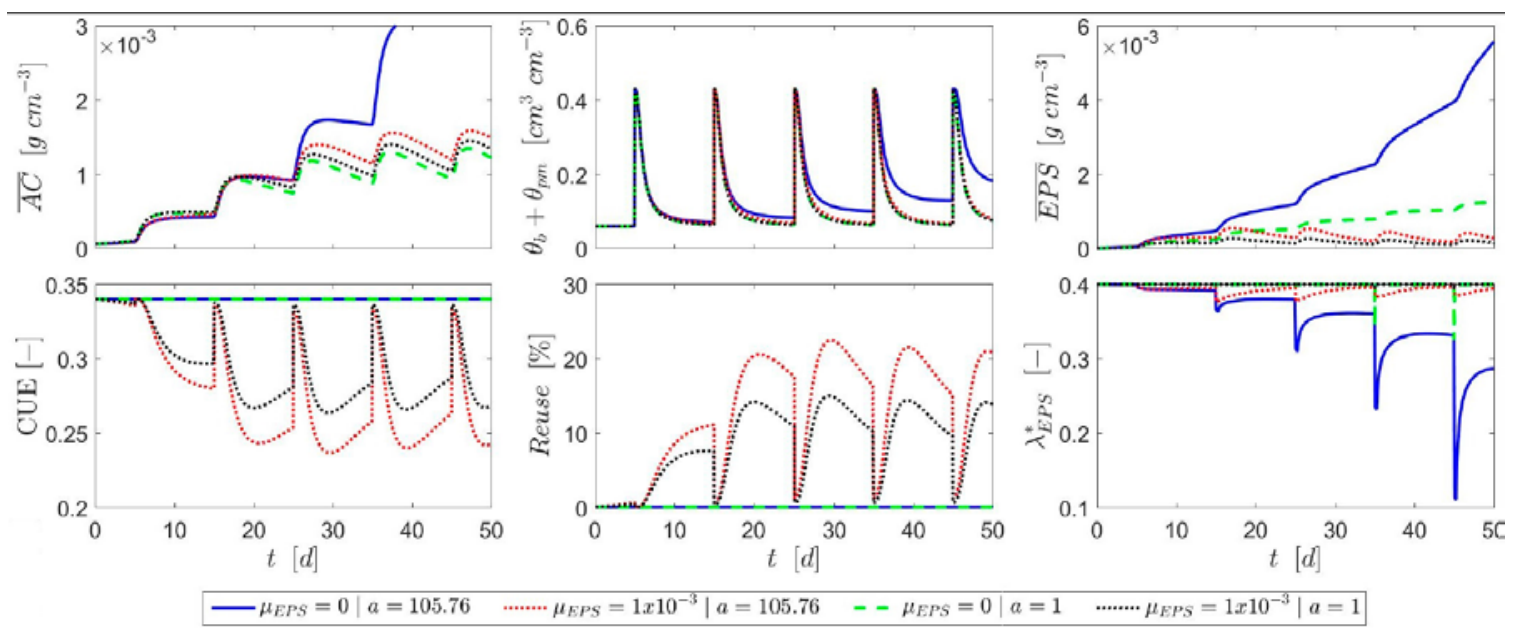

Icaption\{Dynamics of a microbial colony growing in a soil affected by rainfall events with \$ Delta $t \_\{R\}=10 \$$ days, assuming contrasting EPS properties that the effectiveness of their reuse as a source of 
carbon: different decomposition rate capabilities (denoted by the maximum specific decomposition rate $\$ \backslash m u \_\{E P S\}=0 \$$ and $\$ \mid m u \_\{E P S\}=1 \$ x \$ 10^{\wedge}\{-3\} \min ^{\wedge}\{-1\} \$$ ) and EPS water retention capacity $(\$ a=105.76 \$$ and $\$ 1 \mathrm{~cm} \$$ ). Multiple plots show the dynamics of the active cell population $\$ \mid$ overline $\{A C\}$, the total water content \$|theta_\{b\}+|theta_\{pm\}\$, the EPS concentration \$loverline\{EPS\}\$, the carbon-use efficiency (CUE $=A C$ growth over equivalent DOC consumed), the EPS reuse ratio, and the effective coefficient of carbon allocation $\$ \backslash$ lambda_\{EPS $\}^{\wedge}\left\{{ }^{*}\right\} \$$. $\}$

For the studied set of parameters, it was found that when $\$ \mid m u \_\{E P S\}<<1 \$ x \$ 10^{\wedge}\{-3\}$ $\mathrm{g} \$ \mathrm{~cm}^{\wedge}\{-3\} \$$, the EPS production clearly dominated over the reuse rates, resulting in EPS accumulation for any water retention capacity (solid blue and dashed green curves in Figure \ref\{figSM5\}). In contrast, for larger reuse rates, EPS tended to be depleted by consumption, eventually reaching a periodic asymptotic value. The benefits of such an EPS accumulation to the microbial community emerged from the competition between antagonistic properties of EPS: water retention versus carbon storage capacity. On one hand, as already shown before, large EPS concentrations entailed a mitigation of the adverse effects of rains. On the other hand, the carbon stored in EPS could be used as a temporary source of carbon. When these EPS were consumed, the carbon feeding the colony increased, but at the same time, \$loverline\{EPS\}\$ and therefore their capacity to mitigate stresses decreased. The sign of the impact depended then on the combination of parameters: the own compositional characteristics of the EPS (denoted by $\$ a \$)$, their capacity to be reused (\$Mu_\{EPS\}), and again on the environmental circumstances (mainly water inputs). $\ \backslash$

Results in Figure \refffigSM5\} show that only with low EPS retention capacities, the reuse of EPS slightly increased the active biomass (compare dashed green and dotted black curves), because the carbon surplus in EPS helped compensating the dilution and the carbon lost after rainfall. However, when the modeled EPS had a large capacity to retain water, the largest \$loverline $\{A C\} \$$ was obtained when reuse was disabled. The EPS with the lowest retention properties allowed larger potential of carbon accumulation in EPS, because the production rates (characterized by $\$ \backslash \backslash$ lambda^\{*\}_\{EPS\}\$, recall (\ref\{eqSM15\}) and (\ref\{eqSM1\})) remained high, even though relatively large \$ \overline\{EPS\}\$ were achieved. Even though the EPS reuse could improve microbial fitness under specific conditions, it was a rather inefficient 
mechanism in terms of carbon use. The synthesis of biomass by the reuse of EPS required a double respiration cost: for synthesizing EPS first (CUE $\$=Y \$$ ) and for using them to produce new biomass (dotted lines show efficiencies of DOC conversion to biomass lower than \$Y\$). $\ \backslash$

These results suggest that some organisms may use bioengineering design to produce substances of specific composition in order to improve their fitness. We thus speculate that biofilm characteristics could be adapted to environmental conditions to which they are more frequently exposed. For instance, the microorganisms benefitting from EPS reuse may promote large \$Mu_\{EPS\}\$ values by synthetizing enzymes that are efficient at taking carbon from EPS or releasing EPS that are easily decomposed. In a similar way, microbes could adapt the composition of the released compounds to facilitate their later reuse. Lastly, the combination of reuse and reallocation of decay products, which may be induced by cells through the values of $\$ K K_{-}\{D\}^{\wedge}\{A C\} \$$ and $\$ \backslash$ lambda_ $\{d\} \$$, results in a cycle of full carbon reallocation. This process would allow the model to produce any microbial compartment (prioritizing those that are most useful under the actual circumstances), with lower dependence on the external availability of carbon sources.

\section{\subsection\{The Effect of Dormancy on Microbial Dynamics\}}

The capacity to switch in and out of the dormancy state was evaluated by comparing the dynamics of non-EPS producer microorganisms unable to become dormant (\$ $\$ tau_\{i\}=0\$, dashed curves in Figure \refffigSM6\}) with different switching rates between the two states $\left(\$ \backslash\right.$ tau_ $\{i\}=1.32 \$ x \$ 10^{\wedge}\{-4\} \$$ and $\$ 1.32 \$ x \$ 10^{\wedge}\{-3\} \min ^{\wedge}\{-1\} \$$, depicted by dotted and solid curves, respectively). The reactivation rate $\$ \backslash t a u \_\{a\} \$$ was set equal to \$1.43/tau_\{i\}\$ \citep[based on][]\{Konopka1999\}. The switch to a dormant stage was triggered by artificially changing the \$loverline $\{D O C\} \$$ in a fully saturated soil from $\$ 1 \$ \mathrm{x} \$ 10^{\wedge}\{-6\} \$$ to $\$ 0 \mathrm{~g} \$ \$ \mathrm{~cm}^{\wedge}\{-3\} \$$, which modulated the effective transition rates via the coefficient $\$ \mid x i \_\{D O C\} \$$. This approach, based on resource availability, is conceptually (but not mathematically) similar to previous models of activity \citep\{Stolpovsky2011\} or physiological state \citep\{Blagodatsky1998\}. Other models assume that dormancy is instead triggered by a change in soil water content 
Icitep\{Bar2002\} or by water potential \citep\{Manzoni2014\}. The latter approach assumes that transition to dormancy is not caused by carbon starvation \textit\{per se\}, but rather by accumulation of osmolytes or loss of turgor pressure. These alternative approaches (dormancy driven by carbon starvation vs. hydric stress) give similar results in dry soils, where carbon availability is reduced by transport limitations. However, predictions diverge in wet scenarios, where carbon may be limited by dilution, indicating that inactivation occurs only in models in which dormancy is triggered by low carbon availability. \I

At the early stage of biofilm colonization, with moderately high carbon availability

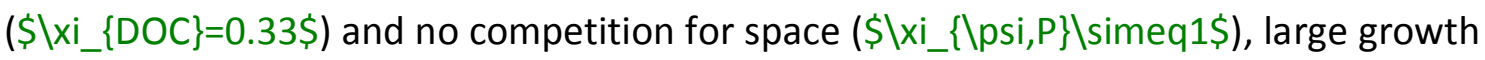
rates were obtained (all curves in Figure \ref\{figSM6\}). Those microorganisms with lower inactivation capacity (\$tau_\{i\}\rightarrow0\$) grew faster (dashed lines). When \$ $\backslash$ tau_ $\{i\}>0 \$$, since $\$ \mid x i \_\{D O C\} \backslash n e q 1 \$$, some of the newly synthetized cells switched to a dormant state (dotted red and solid black curves). This phenomenon led to a delay in the initial growth of the $A C$ pool and in the total amount of cells (\$overline $\{A C\}+\backslash$ overline $\{D C\} \$$ ). As a consequence, the inactivation process seemed apparently counterproductive. After this initial transient stage, the total biomass reached similar values during periods of abundant carbon regardless of their dormancy capacity, even though the colonies with larger \$ $\backslash$ tau_\{i\}\$ had smaller active populations. When the cell number reached their maximum (characterized by low \$ $\mid x i \_\{\mid p s i, P\} \$$, in ( $\backslash$ ref $\{$ eqSM7\})), microbial activity decreased and therefore the amount of carbon consumed increased more slowly. For this set of conditions, the maximum consumption rate was observed at $\$$ |overline $\{A C\} \backslash$ approx $0.2 \mathrm{~g} \$ \$ \mathrm{~cm} \wedge\{-3\} \$$. Above this population, the cellular metabolism was adapted to meet maintenance rather than growth. $\$

When DOC conditions deteriorated at \$Delta t\$ days (\$\|overline $\{\mathrm{DOC}\} \$$ and therefore $\$ \backslash x i \_\{D O C\} \$$ decreased to $\left.\$ 0 \$\right)$, the beneficial effect of dormancy became apparent. Those organisms that switched more rapidly to a dormant state experienced lower losses and maintained significantly larger total cell densities, despite a faster $\$$ \overline $\{A C\} \$$ decrease is observed (compare the dynamics of dashed blue and solid 
blacks lines in Figure \ref\{figSM6\}). Lower mortality rates in DC moderated the total population decline, conferring larger survival chances. \I

When conditions of high DOC availability were restored again (at \$2\Delta t\$, \$4\Delta t\$...), organisms rapidly proliferated. In line with the discussion provided in Icitet\{Blagodatskaya2013\}, respiration increased exponentially after substrate input, reaching its maximum in less than a day and then decreased sharply when the maximum amount of cells was reached or when substrate was removed. Upon DOC removal, the $A C$ pool was fed by the reactivation of $D C$ (if available), reducing the synthesis of new cells (and the carbon consumed). As a consequence, dormancy can be considered an efficient strategy to recycle energy and carbon, allowing lower carbon consumption to achieve the same biomass (bottom panels in Figure \ref\{figSM6\}). 


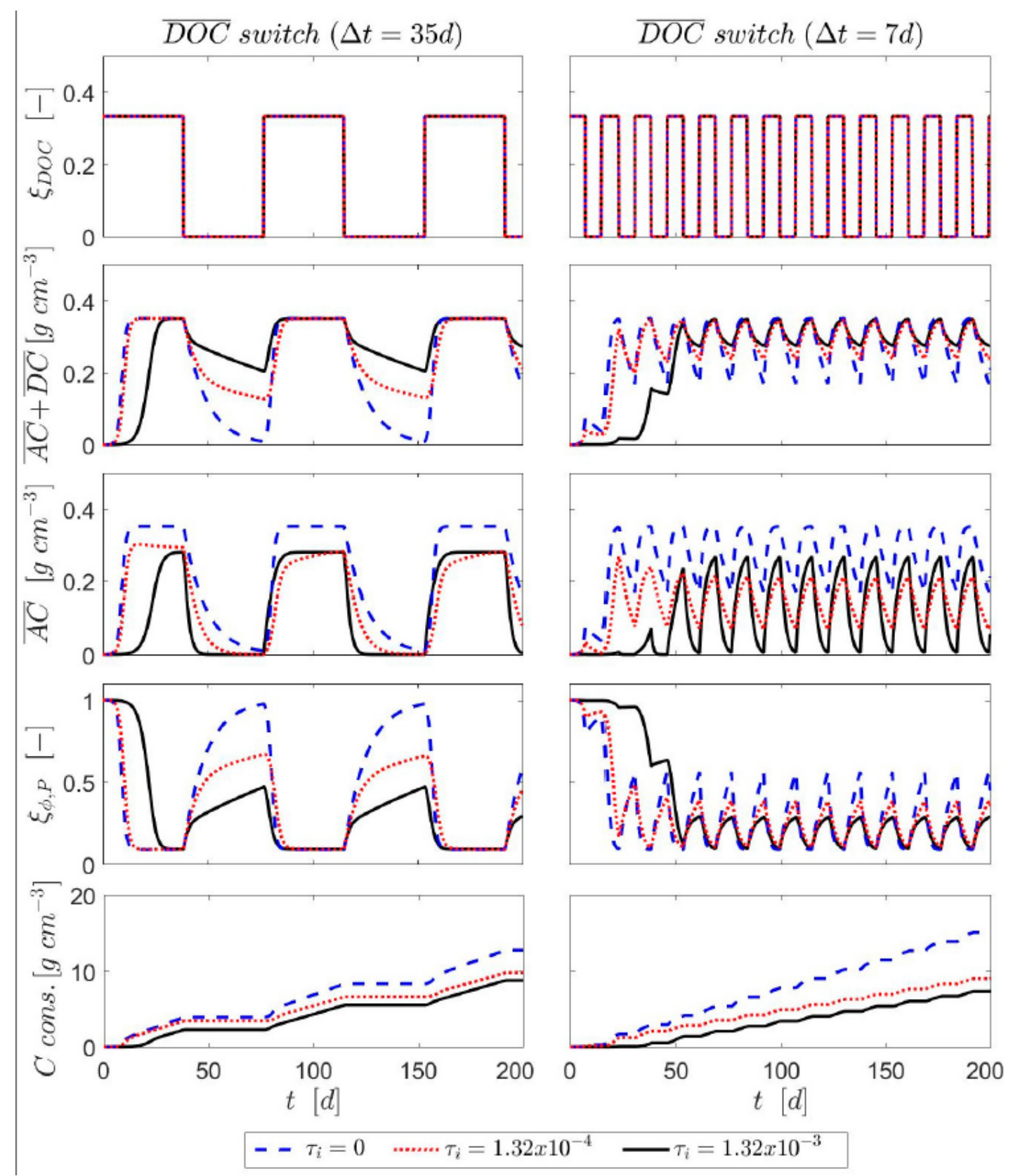

Icaption\{Temporal evolution of biofilm growing in a fully saturated soil that experiences sudden changes in the concentration of DOC every $\$ 35 \$$ (left) and $\$ 7 \$$ days (right). The capacity of switching in and out of a dormant state was evaluated by studying microorganisms unable to become dormant (maximum rate of cell inactivation $\$ \backslash$ tau_\{i\}=0\$) and others with different switching rates between the two states (\$ $\mid$ tau_ $\{i\}=1.32 \$ \times \$ 10^{\wedge}\{-4\} \$$ and $\left.\$ 1.32 \$ \times \$ 10^{\wedge}\{-3\} \quad \min ^{\wedge}\{-1\} \$\right)$. \$|tau_\{a\}\$ was set equal to $\$ 1.43 \$ \times \$ \mid t a u \_\{i\} \$$. From top to bottom: environmental coefficient representing DOC availability (\$\i_\{DOC\}\$), total amount of cells (\$overline $\{A C\}+\backslash$ overline $\{D C\} \$$ ), active cells (\$overline $\{A C\} \$$ ), quorum sensing coefficient based on the cell population (\$ $\mid x i_{-}\{\mid p s i, P\} \$$ ), and cumulative amount of carbon consumed per $\$ \mathrm{~cm}^{\wedge}\{3\} \$$ of soil (C cons.). $\}$

\section{\subsection\{The Combined Effect of Dormancy and EPS on Microbial Dynamics\}}


The combined effect of EPS and dormancy was studied by analyzing the long term concentration of biofilms with different allocation coefficients $\$ \backslash$ lambda_\{EPS $\}=0 \$$ and $\$ \backslash$ lambda_\{EPS $\}=0.23 \$$. Three different scenarios are shown in Figure \ref $\{$ figSM7\}: wet-dry events with $\$ \backslash$ Delta t_ $\{R\}=100 \$$ and $\$ \backslash D e l t a t \_\{R\}=10 \$$ days, and permanent saturation. \\

Results showed that those organisms with larger rates of transition to and from dormancy achieved larger populations in all scenarios after $\$ 1 \$$ year of simulation, regardless of the capacity to produce EPS (top and bottom panels of Figure \ref\{figSM7\}). This indicated that such a mechanism may improve survival chances under a wide range of environmental conditions. Even though the amount of $A C$ in permanently wet scenarios was almost not altered by the inactivation capacity of cells, the total amount of cells experienced a \$26\\% increase (all DC). Particularly, under alternating wet-dry conditions, those biofilms with $\$ \backslash$ tau_\{i\} $\backslash$ geq $1 \$ \times \$ 10^{\wedge}\{-5\} \min ^{\wedge}\{-1\} \$$ increased their total population by $\$ 10 \$$ to $\$ 100 \$$ times compared with the nondormant. Moreover, these colonies had up to a $\$ 70 \$-\$ 95 \backslash \% \$$ of inactive cells (hardly observable in the top plots). The more intense was the water stress, the greater was the benefit resulting from inactivation, being the percentage of DC strongly determined by the environmental constraints. The ratios between active and inactive populations obtained are hardly comparable with the wide range of values reported in the literature \citep[e.g., in the review by][]\{Blagodatskaya2013\}. Results from a sensitivity analysis indicated that other parameter choices could certainly align simulations with alternative empirical evidence. \I 


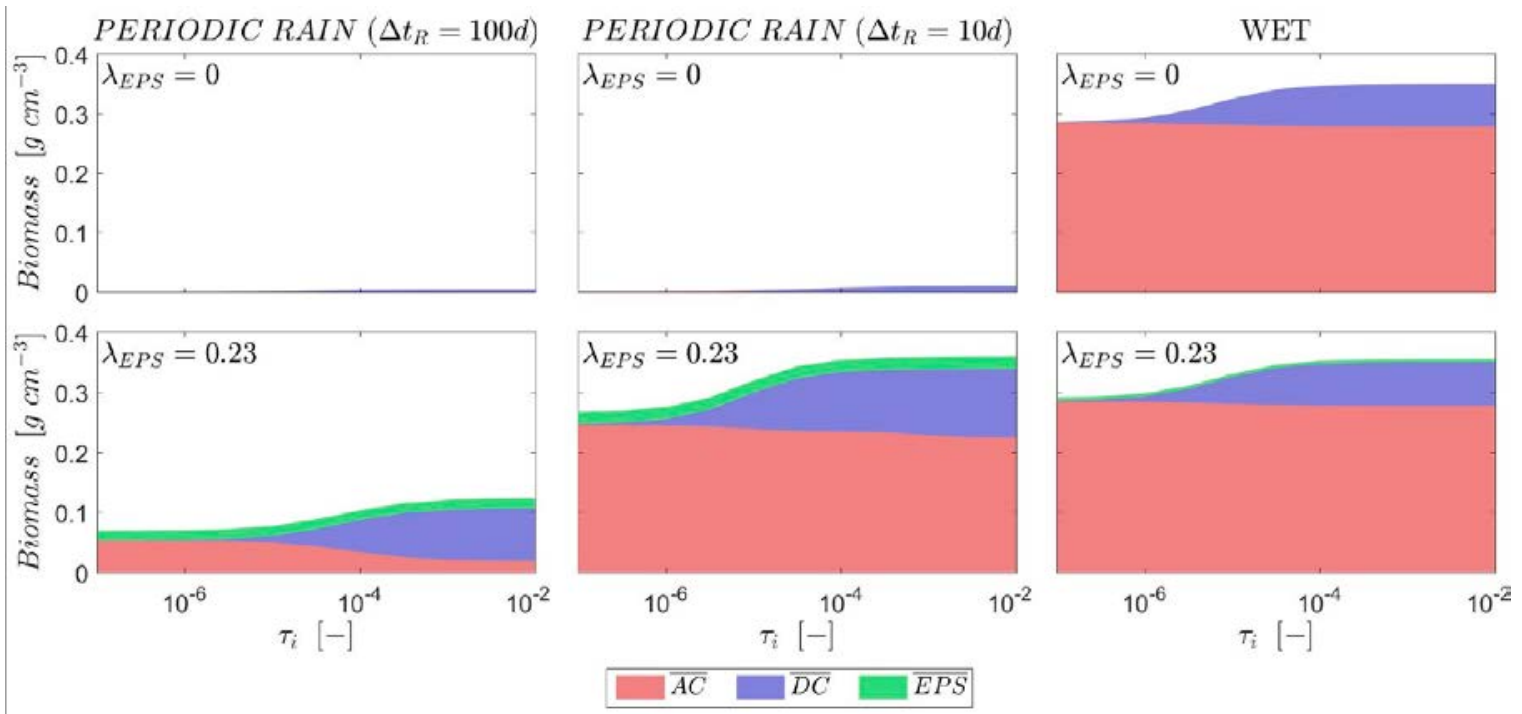

Icaption\{Mass of the biofilm compartments as a function of the maximum rate of cell inactivation (\$tau_\{i\}\$) . Long-term simulations (of \$1\$ year) considered values of \$ $\mid$ tau_\{i\}\$ ranging from $\$ 1 \$ x \$ 10^{\wedge}\{-7\} \$$ to $\$ 1 \$ x \$ 10^{\wedge}\{-2\} \min ^{\wedge}\{-1\} \$$. The role of EPS was evaluated by comparing the results obtained by the environmental coefficient $\$ \backslash$ lambda_ $\{E P S\}=0 \$$ (top row) and $\$ \backslash$ lambda_ $\{E P S\}=0.23 \$$ (bottom row). Simulations shown were performed under periodic rainfall events with \$Delta t_\{R\}=100\$ days (left), \$10\$ days (center), and wet conditions (right). $\}$

Moreover, the additional accumulation of EPS improved microbial fitness, while preserving the positive impact of inactivation. The combined effect of dormancy and EPS production in scenarios affected by periodic rainfall events helped mitigating the carbon and water stress (low saturation and dilution), producing a population rise between $\$ 2-\$$ to $\$ 100 \$$-fold. For the studied set of conditions, the stress remission was so efficient that the cell population reached values comparable to those achieved without stress (see Figure \ref\{figSM7\}, bottom center). $\ \backslash$

Figure \refffigSM8\} depicts contour plots of the long-term concentrations of the AC, $A C+D C$ and EPS compartments as a function of \$Vlambda_\{EPS\}\$ and \$ $\mid$ tau_\{i\}\$. This figure summarizes how combining the release of EPS (\$\lambda_\{EPS\}) and the capacity of organisms to be induced into dormancy (\$)tau_\{i\}\$) had significant effects on the carbon partition in the biofilm. Results show that the growth of biofilms under fully-saturated conditions was not much affected by $\$ \backslash$ lambda_\{EPS $\}$ and $\$ \backslash$ tau_\{i\}\$ because of the downregulation effect and the suitability of the environmental conditions (bottom panels). The inactivation of cells generated colonies with slightly 
larger amounts of cells, while the population of active microbes remained almost stable. It is worth remembering that oxygen shortcomings that may occur frequently in saturated environments are not studied in these simulations. In contrast, biofilm proliferation was limited in environments alternating wet and dry conditions (top panels), particularly common in shallow natural soils, due to the nature and intensity of stressors. Under such circumstances, results confirmed that the combination of the two mechanisms could efficiently counteract the effects of stress, producing a clear improvement of biofilm fitness and microbial survival (as evident from higher \$overline $\{A C\}+\backslash$ overline $\{D C\} \$$ in the top central panel). $\ \backslash$

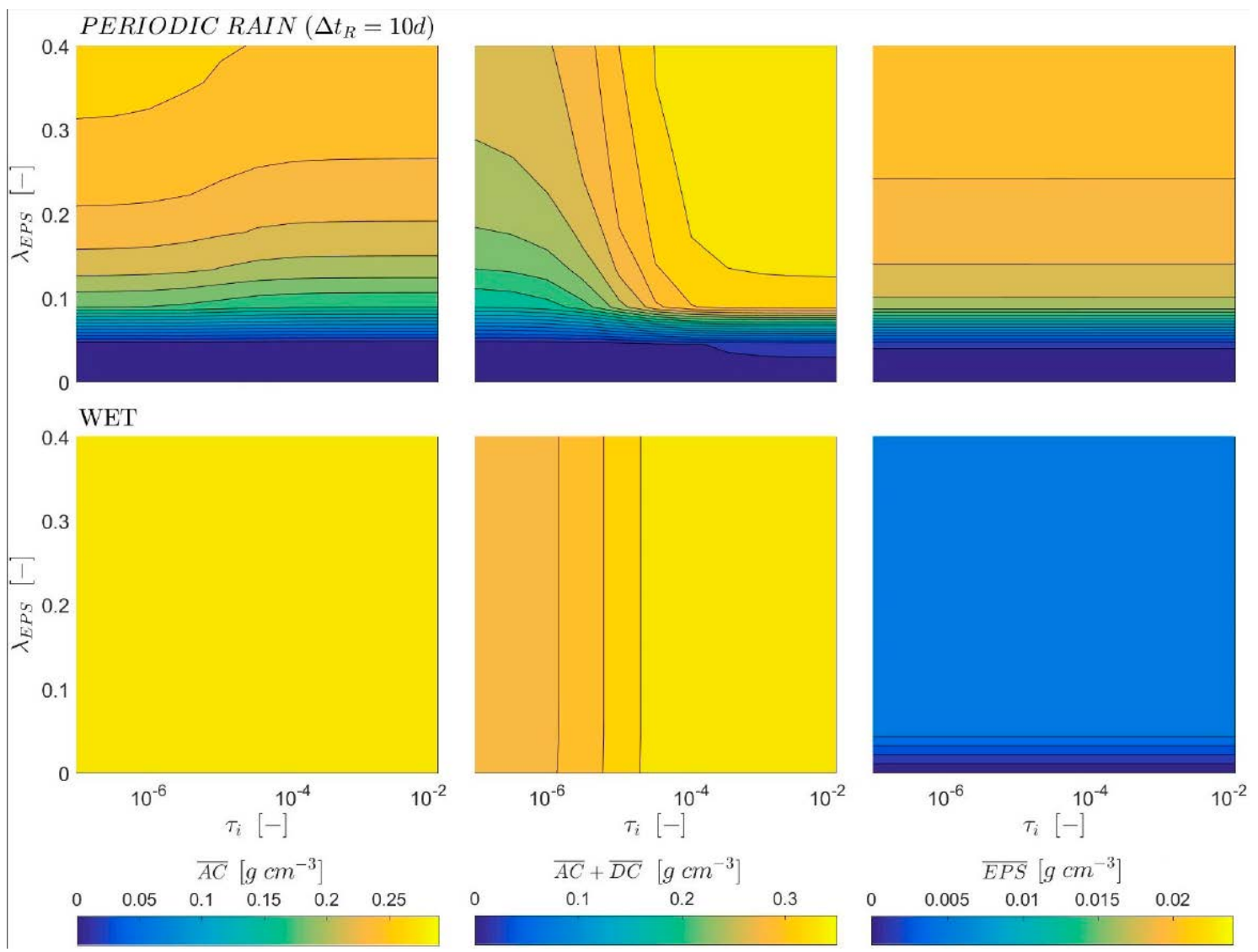

Icaption\{Contour plot depicting the averaged concentrations of biofilm compartments after a long-term simulation of $\$ 1 \$$ year. (left: active cells (\$loverline $\{A C\} \$$ ), center: total cells (\$loverline $\{A C\}+\backslash$ overline $\{D C\}$ ), right: EPS (\$\overline\{EPS\}\$). Simulations were performed for the range of values for the maximum coefficient of carbon allocation towards EPS (\$\lambda_\{EPS\}\$) from $\$ 0 \$$ to $\$ 0.4 \$$ and for the maximum rate of cell inactivation (\$)tau_ $\{i\} \$$ ) from $\$ 1 \$ \times \$ 10^{\wedge}\{-7\} \$$ to $\$ 1 \$ \times \$ 10^{\wedge}\{-2\} \min ^{\wedge}\{-1\} \$$, and under periodic rainfall events with $\$ \mid D e l t a t \_\{R\}=10 \$$ days (top), and wet conditions (bottom).\} 
Based on these results, the significant differences in biofilm composition reported in the literature \citep[e.g.,][]\{Chenu1995,Blagodatskaya2013\} could be attributed to the adaptation of microbes to the heterogeneous conditions in their habitats, in terms of substrate availability, mean saturation and temporal distribution of wetting events.

\section{Isection\{Conclusions\}}

A micro/meso-scale mechanistic model to predict patterns of microbial dynamics under a wide range of conditions (SMMART) is presented and analyzed. This general model differentiates the microbial mass into active and dormant cells, EPS, and extracellular enzymes; and the substrate between particulate and dissolved organic matter. This approach allows disentangling the consequences of contrasting stress response strategies in microbial communities, including synthesis (and reuse) of EPS, transition to a dormant state, and dynamic allocation of carbon among microbial compartments. These strategies are modulated according to biological needs and competition for space, water and carbon substrates in a porous environment. For this purpose, the model is equipped with: (i) indicators that allow the microorganisms to monitor environmental and biological factors, and react accordingly to the current pressures; and (ii) the pore-scale feedbacks existing between biofilm accumulation, carbon availability, and the water retention capacity in bio-amended soils. \I

The performed simulations are used as an explorative tool to elucidate the mechanisms behind observed patterns. Results show that microorganisms may utilize 'smart bioengineering' designs to improve their fitness by adapting the physiological response to the intensity and type of stress. On one hand, the composition and amount of EPS can be readjusted to mitigate preferentially water stress or carbon limitation. First, if EPS has a high water retention capacity, the investments in their production are rewarded by increasing both the overall water content and the carbon availability of soils. Second, those EPS with lower water retention capacity may be used to more competently store the surplus of carbon acquired when conditions are favorable, which can be used at later times if conditions worsen. Third, the amount of EPS produced is modulated according to microbial needs, which are adapted to environmental circumstances. On the other hand, microorganisms able to switch 
between active and dormant states are more efficient in terms of carbon use and survival. The combination of these two strategies provides a clear benefit to colonies in environments affected by alternate wet-dry cycles, which are predominant in soils in most ecosystems. \I

The validation of this model cannot be accomplished easily due to the lack of experimental data and of suitable and non-disruptive techniques to sample the pools considered, and to experimentally characterize some of the parameters included. Moreover, the feedbacks between bioclogging, water flow, detachment, and nutrient transport are not considered even though they might moderately change the conclusions drawn. Nevertheless, despite the assumptions made in the simulations, the most relevant microbial processes involved have been included and the results obtained are qualitatively in agreement with a number of experimental observations reported in the literature. The model can thus be employed as a tool for theoretical exploration and for generating new hypotheses concerning the drivers of biofilm dynamics and their responses to environmental conditions. With slight modifications, this framework could also be applied to non-porous environments such as surfaces in aquatic ecosystems. 